\title{
A Psychometric Network Perspective on the Validity and Validation of Personality Trait Questionnaires
}

\author{
Alexander P. Christensen ${ }^{1 *}$, Hudson Golino ${ }^{2}$, and Paul J. Silvia ${ }^{1}$
}

\begin{abstract}
This article reviews the causal implications of latent variable and psychometric network models for the validation of personality trait questionnaires. These models imply different data generating mechanisms that have important consequences for the validity and validation of questionnaires. From this review, we formalize a framework for assessing the evidence for the validity of questionnaires from the psychometric network perspective. We focus specifically on the structural phase of validation where items are assessed for redundancy, dimensionality, and internal structure. In this discussion, we underline the importance of identifying unique personality components (i.e., an item or set of items that share a unique common cause) and representing the breadth of each trait's domain in personality networks. After, we argue that psychometric network models have measures that are statistically equivalent to factor models, but suggest that their substantive interpretations differ. Finally, we provide a novel measure of structural consistency, which provides complementary information to internal consistency measures. We close with future directions for how external validation can be executed using psychometric network models.
\end{abstract}

Keywords: network analysis, personality, validity, measurement, assessment

Compiled: April $3^{\text {rd }} 2020$

Note: This is the pre-copyedit version of this article, which has been accepted as a part of the European Journal of Personality's special issue on "New approaches towards conceptualizing and assessing personality."

\section{Introduction}

What are personality traits? Your answer likely implies certain hypotheses about the existence of traits and their underlying data generating mechanisms. These hypotheses are usually supported by your choice of psychometric model (Borsboom, 2006). Psychometric models come with a number of assumptions such as how traits cause variation in your measures and the meaning of scores derived from these measures (Borsboom, Cramer, Kievit, Scholten, \& Franic, 2009; Cramer, 2012). These models also come with a number of other consequences such as considerations about how scales should be developed and validated.

For many researchers, personality traits are complex systems-that is, traits are systems in that they are comprised of many components which interact with one another and complex in that their interactions with other systems are difficult to derive because of their dependencies and properties. Despite this view, personality traits are usually not modeled this way. Most psychometric models provide parsimonious perspectives on personality traits, which may arbitrarily carve joints into the fuzzy nature of personality. In addition, these models have causal implications that some researchers might not agree with. Therefore, there is a need for models that better align with how researchers think about personality.

One promising model comes from the emerging field of network psychometrics (Epskamp et al., 2018a). Psychometric network models have a simple representation: nodes (circles) represent variables (e.g., questionnaire items), and edges (lines) represent the unique associations (e.g., partial correlations) between nodes (Epskamp \&
Fried, 2018; Epskamp et al., 2018b). This representation supports the theoretical perspective, often referred to as the network approach (Borsboom, 2008, 2017), that psychological attributes are complex systems of observable behaviors that dynamically and mutually reinforce one another (Schmittmann et al., 2013).

From this perspective, personality traits resemble an emergent property of the interactions that occur between unique behavioral components - that is, traits are not any single component of the system but rather a feature of the system as a whole (Baumert et al., 2017; Cramer et al., 2012a). This suggests that traits emerge because some characteristics and processes within individual people tend to covary more than others (Mõttus \& Allerhand, 2017), and when these relevant processes are aggregated, they reflect meaningful differences between people in the population (e.g., trait domains; Borkenau \& Ostendorf, 1998; Cramer et al., 2012a).

Such an explanation of traits affords a novel context for how they should be assessed. First, it implies that behaviors that are associated with one trait may directly influence behaviors of another trait-the lines separating traits are more fuzzy than they are distinct (e.g., comorbidity in psychopathology; Cramer, Waldrop, van der Maas, \& Borsboom, 2010).

\footnotetext{
${ }^{1}$ University of North Carolina at Greensboro, Greensboro, NC, USA

${ }^{2}$ University of Virginia, Charlottesville, VA, USA

* Corresponding author: alexpaulchristensen@gmail.com
}

How to cite: Christensen, A. P., Golino, H., \& Silvia, P. J. (in press). A psychometric network perspective on the validity and validation of personality trait questionnaires. European Journal of Personality. https://doi.org/10.31234/osf.io/ktejp 
Second, there is an emphasis on a trait's components as much as the trait itself-a trait's observable components do not measure the trait but are instead part of the trait (Schmittmann et al., 2013). This suggests that a behavior such as liking to go to parties is only one part of a causal collection of behaviors that we call extraversion (Borsboom, 2008; Cramer, 2012). This represents a re-focusing on what parts of a trait are being measured rather than the premise that the trait itself is being measured. Finally, this explanation proposes that the behavioral components of a trait are unique, meaning they have distinct causes (Cramer et al., 2012a). This suggests that there should be a shift in how we model existing questionnaires where many related items are often used to measure a single attribute.

The intent of this paper is to elaborate on what the novel perspective provided by the network approach means for personality measurement and assessment. We focus specifically on the validity and validation of personality trait questionnaires with the goal of demonstrating how psychometric network models relate to modern psychometric perspectives. We place a particular emphasis on the structural analysis of validation (e.g., item analysis, dimension analysis, internal structure; Flake, Pek, \& Hehman, 2017; Loevinger, 1957). Before discussing validation, we first consider what it means for a questionnaire to be valid - a topic that has a defining role in the substantive interpretation of personality measures.

\section{(Test) Validity of Personality Trait Question- naires}

The trait approach has a long tradition in personality, significantly shaping the last 30 years of research. Many contemporary theories of personality are inclined to accept traits as phenomena that exist in some form (e.g., concrete biological entities, abstract population summaries). Across theories, traits seek to provide parsimonious descriptions of broad between-person patterns of covariation at the population-level (Baumert et al., 2017). Five or six higherorder traits are commonly thought to represent the majority of these between-person differences, which are typically assessed using questionnaires (Lee \& Ashton, 2004; McCrae $\&$ Costa, 1987). The validation of these questionnaires is a critical part of the research agenda (Flake et al., 2017).

There are many views on what validity means with the most common perspectives involving the interpretation of test scores (e.g., Cronbach \& Meehl, 1955; Kane, 2013; Messick, 1995). This is not how we view validity; instead, we adopt the definition that validity refers to whether a test measures what it intends to measure (Borsboom et al., 2009; Cattell, 1946; Kelley, 1927). Borsboom and colleagues (2004) refer to this as test validity, which states that "a test is valid for measuring an attribute if and only if (a) the attribute exists and (b) variations in the attribute causally produce variations in the outcomes of the measurement procedure" (p. 1061). An attribute refers to a property that exists prior to and independent of measurement (Loevinger, 1957). This definition of validity involves connecting the structure of an attribute to the response processes of a measure.

In this section, we provide an overview of what this definition means for the validity of personality trait questionnaires. Most of the heavy lifting for the relation between a personality trait and questionnaire is done by a researcher's choice of psychometric model (Borsboom, 2006). The most common model used for validation in the personality literature is the latent variable model (Flake et al., 2017). Therefore, we begin our discussion of validity by briefly reviewing how latent variable models make sense of the test validity criteria (for a more through treatment, see Borsboom, Mellenbergh, \& van Heerden, 2003). We then move to psychometric network models, which have emerged as an alternative explanation for the coherence of traits. In terms of validity, much less has been put forward for psychometric network models and personality questionnaires; therefore, we spend most of this section reviewing its current state and discussing its meaning in the context of personality measurement. Throughout this section, we refer to a hypothetical questionnaire of extraversion to contextualize our points.

\section{Latent Variable Perspective}

In personality (and most of psychology), reflective latent variable models, where the indicators are regressed on the latent variable (i.e., causal arrows point from the latent variable to the indicators), are the standard conceptualization of measurement (Borsboom et al., 2003). A reflective latent variable model holds that the items in our questionnaire are a function of the latent variable, meaning that people's responses to our questionnaire are caused by their position on the latent variable (e.g., extraversion). Using this causal explanation, we can evaluate the criteria for test validity.

First, does the attribute extraversion exist? That is, does extraversion exist prior to and independently of our questionnaire? Many researchers would consider this question trivial; however, to maintain that extraversion is indeed an attribute that exists, then the latent variable must be causally responsible for the responses to our questionnaire (Borsboom et al., 2003). Indeed, this is how many researchers think about the relationship between personality traits and their questionnaires as well as what some theories of personality suggest (McCrae \& Costa, 2008; McCrae et al., 2000).

The second criterion is the crux of test validity which, as Borsboom and colleagues (2004) point out, is not so straightforward. This is because it requires a theory for how extraversion can be linked to the response processes of our questionnaire. Difficulties arise because it's plausible (and even likely) that the processes that lead one person to respond with "agree" to an item (e.g., "I like to go to parties") and another person to respond "agree" to the same item are different (Borsboom \& Mellenbergh, 2007). An idealistic perspective is that people have different response processes but they select "agree" on the same item because they are positioned similarly on extraversion. With this perspective, a common and implicit interpretation is that people possess some quantity of extraversion and it's the difference in these quantities that cause the variation in how people respond to our questionnaire. More simply, Alice scores higher on our questionnaire than Bob because Alice is positioned higher on the extraversion continuum than Bob.

From a causal perspective, a defensible account for how extraversion causes variation in our questionnaire would be that "population differences in position on [extraversion] 
cause population differences in the expectation of item responses" (Borsboom et al., 2003, p. 211). This implies that people in the population which occupy the same position on extraversion will typically respond similarly to the same items in our questionnaire. This brings us back to the first criterion: Does extraversion exist? Or rather, to what extent does extraversion exist? One claim would be that extraversion exists as a between-person attribute - that is, it is a population attribute. A population attribute is not necessarily possessed by any one person in the population but rather represents between-person differences at the population-level (Baumert et al., 2017; Cervone, 2005). This notion aligns well with the Allportian view that "[a] common trait is a category for classifying functionally equivalent forms of behavior in a general population of people" (Allport, 1961, p. 347).

From a non-causal perspective, this means that extraversion could exist as a useful descriptor for comparing people rather than explaining their behaviors (Hogan \& Foster, 2016; Pervin, 1994). Many personality researchers hold this view of reflective latent variables (e.g., Ashton \& Lee, 2005; Goldberg, 1993). Therefore, researchers need not view a reflective latent variable as causal but rather as a summary statistic of the shared variance between items in our questionnaire. This leaves our questionnaire's validity as a subject of substantive interpretation - that is, it depends upon what researchers think they are measuring: traits as population-level positions or traits as descriptive summaries of between-person differences in the population.

\section{Psychometric Network Perspective}

Psychometric network models have been proposed as an alternative explanation for the emergence of personality traits. From a psychometric network perspective, traits arise not because of a latent common cause but rather from the causal (bi)directional relationships between observed variables (Cramer et al., 2012a). This explanation suggests that latent traits are not necessary to explain how items in our questionnaire covary (Borsboom et al., 2009). Moreover, it implies that traits do not exist or at least they do not exist in a classical sense of measurement (i.e., causing variation in our questionnaire; Cramer, 2012). Instead, the relationship between extraversion and our questionnaire is a mereological one-that is, the items in our questionnaire do not measure extraversion but are part of it (Borsboom, 2008; Cramer et al., 2012a).

Extraversion is therefore a summary statistic for how personality components are influenced by one another (e.g., liking to talk to people $\rightarrow$ liking to go to parties $\longleftrightarrow$ liking to meet new people; Cramer, 2012). In this sense, extraversion exists as a state of the network or the stable organization of dynamic personality components that are mutually activating one another (Cramer et al., 2012a; Schmittmann et al., 2013). Our questionnaire thus refers to the state of a specific set of personality components that are causally dependent on one another and form a network (Cramer, 2012). The state of the network is determined by the total activation of these components and is what we refer to as extraversion - that is, the more personality components that are active, the more the network is pushed towards an extraverted state (Cramer, 2012).
In the context of a between-person network model, our questionnaire's network represents the aggregation of the average activation of each component across within-person networks (i.e., each individual person's network across several time points; Cramer et al., 2012a; Epskamp et al., 2018b). Both theory and empirical evidence appear to support this claim. From a Whole Trait Theory perspective (Fleeson \& Jayawickreme, 2015), people's responses to items in self-report questionnaires correspond to their locations and maximums of their density distributions for the respective within-person states (Fleeson, 2001; Fleeson $\&$ Gallagher, 2009). When aggregated, these states tend to correspond to self-reported traits (Rauthmann, Horstmann, \& Sherman, 2019), and when compared across people, these states typically produce the between-person traits (Borkenau \& Ostendorf, 1998; Hamaker, Nesselroade, \& Molenaar, 2007). This interpretation leaves open an important question: What then do personality components refer to?

\section{Personality components}

Cramer and colleagues (2012a) define personality components as "every feeling, thought, or act" that is associated with a "unique causal system" (p. 415). In most instances, these components refer to items of a questionnaire. A key point of emphasis in their definition is that these components are unique in that they are causally autonomous (i.e., distinct causal processes). For many existing personality questionnaires, items are often not unique. Instead, facets or narrower characteristics of a trait (e.g., gregariousness, warmth, assertiveness) are comprised of many closely related and sometimes redundant items. In this sense, personality components that comprise extraversion may be items but they also may be facets (Costantini \& Perugini, 2012).

In our view, this represents a key difference between facets and components: Facets are a collection of related items (not necessarily sharing a common cause), while components are an item or set of items that share a unique common cause. This distinction is important because some facets in existing questionnaires reflect a homogeneous cause (and are therefore considered a component), while other facets reflect heterogeneous causes, which must be separated into unique components. ${ }^{1}$ Therefore, a facet from the network perspective would be a set of unique components that coalesce into a meaningful sub-organization of a trait's domain. From this perspective, it becomes imperative that researchers determine whether items and facets of an existing questionnaire are distinct autonomous causal components or if they reflect a common cause (Hallquist, Wright, \& Molenaar, 2019).

Based on this definition, personality components appear to closely resemble attributes. In this way, extraversion may exist as a composite attribute or an attribute that is comprised of many other attributes (Borsboom \& Mellenbergh, 2007). The number of attributes that constitute extraversion then becomes a function of the sampling properties from its domain of representative attributes (McDonald, 2003). Importantly, the selection of attributes will change the composition of the network,

${ }^{1}$ The use of "reflect" in our language is on purpose and implies a common cause that can be associated with a reflective latent variable. 
meaning that different questionnaires will have different compositions despite still plausibly refering to extraversion (Markus \& Borsboom, 2013). Extraversion should then be viewed as a finite universe of attributes where there are a limited number of unique attributes that can comprise it (McDonald, 2003). Therefore, there is a particular need to identify and validate the content of each personality trait's domain (Markus \& Borsboom, 2013).

It's tempting to say that researchers should only measure attributes that represent one domain; however, it's unlikely that attributes of a personality trait will exist independently of other trait domains (Schmittmann et al., 2013; Schwaba, Rhemtulla, Hopwood, \& Bleidorn, 2020; Socan, 2000). An item like "enjoys talking to people," for example, certainly represents the domain of extraversion but it may also represent the domain of agreeableness. Common examples of this cross-domain entanglement often occur in psychopathological comorbidity (Cramer et al., 2010). Thus, distinctions between what attributes constitute the extraversion domain become rather fuzzy and a matter of degree because of the overlap representative attributes can have with other domains (Schmittmann et al., 2013). Such fuzziness is likely common in personality where attributes may not clearly delineate between where one trait begins and another ends (Connelly, Ones, Davies, \& Birkland, 2014). Indeed, this is exactly what functionalist and complex system theories of personality suggest (Cramer et al., 2012a; Perugini, Costantini, Hughes, \& De Houwer, 2016; Read et al., 2010; Wood, Gardner, \& Harms, 2015) and what recent psychometric network analyses of personality traits find (Schwaba et al., 2020).

\section{Validity from the network perspective}

This leads to the question of how extraversion (as a composite attribute) can cause variation in our questionnaire. Quite simply, it does not: There is no link between the (composite) attribute and the questionnaire's response processes because it does not exist (Schmittmann et al., 2013). This is because no single attribute that extraversion is comprised of will directly assess extraversion; instead, each attribute assesses parts of the extraversion domain (Borsboom, 2008; Borsboom \& Mellenbergh, 2007; Cramer et al., 2012b). With this perspective, we can say that the variation in our questionnaire arises from the sampling of attributes in the representative domain (Borsboom \& Mellenbergh, 2007), which is clear from studies that have examined several different questionnaires (e.g., Christensen et al., 2019; Schwaba et al., 2020).

When evaluating whether our questionnaire is a valid measure of extraversion from the network perspective, we must shift the evaluation from the validity of extraversion as an attribute to the validity of its components. This does not rule out the validity of the questionnaire but rather shifts the perspective such that our questionnaire is measuring the state of the network comprised of causal connected components that we refer to as extraversion. The explanation for the variation of our measurement thus comes from the reciprocal cause and effect of other attributes in the network.

This explanation does not come without consequence. The issue of connecting the attribute to response processes is merely side stepped from personality traits to personality components. The response processes in network models are assumed to lie in the reciprocal cause and effect of other components. Indirectly, this suggests that the responses processes of one component has reciprocal causes and effects on processes of other components. This point, however, is circular in that it still does not specify what the response processes are.

Although the network perspective avoids introducing latent variables to account for these response processes, it does not avoid the question of how they occur. To this end, it is important for personality researchers from the network perspective to connect response processes to personality components. More specifically, researchers must seek to specify how response processes of one component can cause and effect processes of another component. We do not claim to have a definitive answer to this issue, but highlight it as one that is particularly perplexing and requires sophisticated research designs (e.g., Costantini, Richetin, et al., 2015).

\section{Validation of Trait Questionnaires from a Psychometric Network Perspective}

Our discussion of validity to this point has been about whether a questionnaire possesses the property of being valid. This discussion sets up how psychometric evaluations of a questionnaire should be substantively interpreted during validation. Validation differs from validity in that it is an ongoing activity which seeks to describe, classify, and evaluate the degree that empirical evidence and theoretical rationales support the validity of the questionnaire (Borsboom et al., 2004; Messick, 1989). Validation usually entails three phases: substantive, structural, and external (Flake et al., 2017). Our main focus will be on the structural phase, which primarily consists of establishing evidence that our questionnaire measures what we intend it to through redundancy (e.g., item analysis), dimension, and internal structure (e.g., internal consistency) analyses.

Validation from a psychometric network perspective has received relatively little attention. To date, psychometric network models have mainly been used as a novel measurement tool, which has led to an alternative account for the formation of traits (Costantini, Epskamp, et al., 2015; Cramer et al., 2012a). When it comes to psychometric assessment, the scope of psychometric networks has been far more limited (e.g., dimension reduction methods; Golino \& Epskamp, 2017; Golino et al., in press). There does, however, appear to be some potential because networks have been shown to be mathematically equivalent to latent variable models (Guttman, 1953; Kruis \& Maris, 2016; Marsman et al., 2018).

The key to distinguishing network psychometrics models from latent variable models is to establish how the measures of these models differ in their substantive interpretations (i.e., hypothesized data generating mechanisms; van Bork et al., 2019). We will draw on several points from the previous section on validity to elaborate on these interpretations. In the end, the aim of this section is to take the initial steps toward a formalized framework for the use of psychometric network models in the validation of personality questionnaires. 


\section{Overview}

To achieve this aim, we divide this section into three parts, which represent the order in which researchers should proceed with structural validation from the psychometric network perspective. First, we cover the initial phase of redundancy analysis for reducing redundancy in personality questionnaires. Next, we discuss dimension analyses. Within this section, we connect communities and node strength of network models to factors and factor loadings of latent variable models, respectively. Finally, we present a novel measure of internal structure that can be used to assess the extent to which a scale (or dimension) is comprised of a set items that are homogeneous and interrelated in a multidimensional context. ${ }^{2}$

In our discussion, it's important that we make clear that we view network models as complementary to latent variable models and therefore suggest that they can be synergistically leveraged. The main difference between them, as we discussed in our section on validity, is the proposed data generating mechanisms. From a statistical point of view, network models offer additional information about the relationship between variables that are not possible in latent variable models since in the latter the relationship between items are accounted by the factor. Analyses about the structure of the system (e.g., topological analysis), for example, can be implemented in network models, helping researchers uncover important aspects of the system (Borsboom, Cramer, Schmittmann, Epskamp, \& Waldorp, 2011). Because of this, we connect network models to latent variable models (where applicable) and highlight the substantive differences that these models imply. As a general point, we recommend at least 500 cases when performing these analyses, which is based on previous simulation studies (Christensen, 2020; Golino et al., in press).

\section{Redundancy Analysis}

In scale development, a researcher must establish what items to include, which involves determining the desired specificity and breadth of the trait(s) the researcher is trying to measure. Greater specificity leads to scales that have higher internal consistency, which increases the likelihood that the researcher is measuring the same attribute while reducing idiosyncrasies specific to each item (DeVellis, 2017). Greater breadth leads to scales that have higher item-specific variance, which increases the coverage of the representative domain (McCrae, 2015). In many existing trait questionnaires, researchers have focused on achieving a balance of both - that is, some facets reflect a single narrow attribute, while other facets are composites of several attributes.

One recent suggestion for questionnaires aimed at trait domains is to favor breadth in order to maximize information and efficiency (McCrae \& Mõttus, 2019). Based on what we've outlined in our section on validity, psychometric network models align well with this suggestion. Indeed, a key notion of network psychometrics is that personality traits are comprised of unique causal components, meaning the components are not exchangeable with other components of the system (Cramer et al., 2012a). As a consequence, these components should be unique rather than redundant to reduce latent confounding (Hallquist et al., 2019). This implication perhaps marks the biggest validation difference between network and latent variable models.

Because most existing personality scales have been developed from a latent variable perspective, researchers must make careful considerations about using psychometric network models with existing scales because they are likely to have homogeneous facets (Costantini \& Perugini, 2012). Take, for example, the SAPA Personality Inventory (Condon, 2018) where items "Hate being the center of attention," "Make myself the center of attention," "Like to attract attention," and "Dislike being the center of attention" clearly have a common underlying attribute: attentionseeking. From the psychometric networks perspective, these items are not unique components themselves but comprise a single unique component. This makes the first step of questionnaire validation from a psychometric network perspective to identify and handle redundancy in scales.

\section{An approach to statistically identify redundancy}

In the network literature, the network measure, clustering coefficient, has been considered as a measure of redundancy in personality networks (Costantini et al., 2019; Dinic, Wertag, Tomasevic, \& Sokolovska, 2020). A node's clustering coefficient is the extent to which its neighbors are connected to each other, forming a triangle. Although this measure is useful for describing whether a node is locally redundant, it does not provide information about which nodes in particular a target node is redundant with. Here, we conceptually describe an approach to identify whether a node is statistically redundant with other nodes in a network.

Our approach begins by first computing a similarity measure between nodes. One method for doing so is called weighted topological overlap (Zhang \& Horvath, 2005), which quantifies how similar two nodes' connections to other nodes are. More specifically, it quantifies the similarity between the magnitude and direction of two nodes' connections to all other nodes in the network. In biological networks, these measures have been used to identify genes or proteins that may have a similar biological pathway or function (Nowick, Gernat, Almaas, \& Stubbs, 2009). Thus, greater topological overlap suggests that two genes may belong to the same functional class compared to those with less overlap. In the context of a personality network, nodes that have large topological overlap are likely to have shared functional or latent influence. From a more traditional psychometrics perspective, one method would be to identify items that have high residual correlations after the variance of facets and factors have been removed. ${ }^{3}$

Although the weighted topological overlap measure provides numerical values, from no overlap (0) to perfect overlap (1), for each node pair in the network, it does not include a test for significance. In order to determine which node pairs overlap significantly with one another, we apply the following approach. First, we obtain only the values that are greater than zero-node pairs that have a topological

${ }^{2}$ We provide a full walkthrough example of these analyses in the Supplementary Information using R (R Core Team, 2020). Our example uses data that is freely available in the psychTools package (Revelle, 2019) and assesses the five-factor model using the SAPA inventory (Condon, 2018).

${ }^{3}$ We thank the anonymous reviewer who pointed out this possibility. 
overlap of zero are not connected in the network and are therefore not informative for determining significance of overlap. Next, we fit a distribution to these non-zero values using the fitdistrplus package (Delignette-Muller \& Dutang, 2015) in R. The parameters (e.g., $\mu$ and $\sigma$ from a normal distribution) from the best fitting distribution (based on Akaike information criterion) are then used as our probability distribution.

For each node pair with a non-zero topological overlap value, we compute the probability of achieving its corresponding value from this distribution. These probabilities correspond to $p$-values. Using a multiple comparison method, node pairs whose $p$-values are less than the corrected alpha are considered to be significantly redundant. We've implemented this approach in the EGAnet package (Golino \& Christensen, 2020) in R under the function node. redundant (see the Node Redundancy section in the Supplementary Information). Results from one simulation study found that the adaptive alpha multiple comparison correction method (Perez \& Pericchi, 2014) had the fewest false positives and negatives and highest accuracy of all the methods tested (Christensen, 2020).

\section{Options for handling redundant nodes}

This approach provides quantitative evidence for whether certain items are redundant. We recommend, however, that researchers verify these redundancies and use theory to determine whether two or more items represent a single attribute (i.e., a common cause). There are two options that researchers can take when deciding how to handle redundancy in their questionnaire. The more involved option is to remove all but one item from the questionnaire. When taking this option, there are a few considerations researchers must make. Qualitatively, which item represents the most general case of the attribute? Often items are written with certain situations attached to them (e.g., "I often express my opinions in group meetings"; Lee \& Ashton, 2018), which may not apply to all people taking the questionnaire. Therefore, more general items may be better because they do not represent a situation-specific component of an attribute (e.g., "I often express my opinions"). Quantitatively, which item has the most variance? This is a common criterion in traditional psychometrics because greater variation suggests that this item better discriminates between people on the specific attribute (DeVellis, 2017).

The more straightforward option is to combine items into a single variable. This can be done by estimating a reflective latent variable consisting of the redundant items and using the latent scores. We strongly recommend this latter approach because it retains all possible information from available data. We've implemented an interface to manage this second option using the node.redundant.combine function in the EGAnet package in R. We describe how to apply this approach in the Supplementary Information, including heuristics to use when deciding which items are redundant.

\section{Dimension Analysis}

Dimensionality

Dimensionality assessment is an integral step for validating the structure of a questionnaire. The general consensus among researchers is that personality traits are hierarchically organized at different levels of breadth and depth (John \& Srivastava, 1999; McCrae \& Costa, 2008). Usually, trait domains are decomposed into facets, which are further broken down into items (McCrae \& Costa, 1987, 2008). More recently, aspects (between traits and facets) were added to the hierarchy (DeYoung, Quilty, \& Peterson, 2007). Personality questionnaires tend to follow this structure with most assessing multiple domains and facets - there typically are five or six trait domains and for every domain, there are several facets (ranging from two to nine).

In traditional psychometrics, factor models (e.g., EFA) are the most common method used to assess the dimensionality of a trait domain (Flake et al., 2017). In psychometric networks, the main methods used to assess dimensionality of the network are called community detection algorithms (Fortunato, 2010). These algorithms identify the number of communities (i.e., dimensions) in the network by maximizing the connections within a set of nodes, while minimizing the connections from the same set of nodes to other sets of nodes in the network. Rather than these communities forming because of a common cause, psychometric network models suggest that dimensions emerge from densely connected sets of nodes that form coherent sub-networks within the overall network.

Despite these frameworks proposing different data generating mechanisms, the data structures do not necessarily differ (van Bork et al., 2019). Indeed, a researcher can fit a factor model to a data structure generated from a network model with good model fit (van der Maas et al., 2006). Similarly, a network model with a community detection algorithm can be fit to a data structure generated from a factor model and identify factors (Golino \& Epskamp, 2017; Golino et al., in press). This underlying equivalence follows from the fact that any covariance matrix can be represented as a latent variable and network model (van Bork et al., 2019). The statistical equivalence between these models has been well documented (e.g., Epskamp et al., 2018a; Guttman, 1953; Kruis \& Maris, 2016; Marsman et al., 2018). Therefore, factors of a latent variable model and communities of a network model are statistically equivalent (Golino \& Epskamp, 2017).

Indeed, Guttman (1953) demonstrates that there is a direct equivalence between network and factor models. Although network models were not yet specified in the area of psychology, Guttman (1953) proposed a new factor analytic approach termed image structural analysis, which is essentially a network model with node-wise estimation using multiple regression (e.g, Haslbeck \& Waldorp, 2015). Guttman mathematically demonstrated how image structural analysis relates to factor models, and suggested that factor models were a special case of the nodewise network model where the errors of the variables are made to be orthogonal. ${ }^{4}$ Therefore, the difference between

${ }^{4}$ We thank Denny Borsboom for pointing us to the Guttman (1953) paper. 
the models is their suggested data generating mechanisms, which is provided by their visual representations.

It's important that we acknowledge that in some cases, factors of a factor model may represent causally dependent interactions between components (rather than a common cause); in other cases, communities of a network model may represent a common cause (rather than causally dependent interactions between components). Moreover, other explanations could be that external causes such as situational factors (Cramer et al., 2012a; Rauthmann \& Sherman, 2018) or goals and motivations (Read et al., 2010) could lead to personality dimensions.

\section{Exploratory Graph Analysis}

The most extensive work on dimensionality in the psychometric network literature has been with a technique called Exploratory Graph Analysis (EGA; Golino \& Epskamp, 2017; Golino et al., in press). The EGA algorithm works by first estimating a Gaussian Graphical Model (Lauritzen, 1996), using the graphical least absolute shrinkage and selection operator (GLASSO; Friedman, Hastie, \& Tibshirani, 2008), where edges represent (regularized) partial correlations between nodes after conditioning on all other nodes in the network. Then, EGA applies the Walktrap community detection algorithm (Pons \& Latapy, 2006), which uses random walks to determine the number and content of communities in the network (see Golino et al., in press for a more detailed explanation). ${ }^{5}$ Several simulation studies have shown that EGA has comparable or better accuracy for identifying the number of dimensions than the most accurate factor analytic techniques (e.g., parallel analysis; Christensen, 2020; Golino \& Demetriou, 2017; Golino \& Epskamp, 2017; Golino et al., in press).

Beyond performance, EGA has several advantages over more traditional methods. First, EGA does not require a rotation method. Rotations are rarely discussed in the validation literature but can have significant consequences for validation (e.g., estimation of factor loadings; Browne, 2001; Sass \& Schmitt, 2010). For EGA, orthogonal dimensions are depicted with few or no connections between items of one dimension and items of another dimension. Second, researchers do not need to decide on item allocation - the algorithm places items into dimensions without the researcher's direction. For EFA, in contrast, researchers must decipher a factor loading matrix. Third, the network plot depicts some dimensions as more central than others in the network (see Dimensionality section in the Supplementary Information). Thus, EGA can be used as a tool for researchers to evaluate whether the items of their questionnaire are coalescing into the dimensions they intended and whether the organization of the trait's structure is what they intended. Finally, the network plot also depicts levels of a trait's hierarchy as continuous - that is, items can connect between different facets and traits. This supports a fuzzy interpretation of the trait hierarchy where the boundaries between items, facets, and traits are blurred.

With these advantages, it's important to note their similarities to factor analytic methods. For instance, most community detection algorithms used in the literature (including the Walktrap) sort items into single dimensions. This creates a structure that is akin to a typical confirmatory factor analysis (CFA; i.e., items belonging to a single dimension), which constrains the interpretation of a continuous hierarchy. There are, however, algorithms in the broader network literature that allow for overlapping community membership (e.g., Blanken et al., 2018), which may better represent these fuzzy boundaries and how researchers think about personality. Another limitation is that the factor loading matrix of an EFA model can equivalently represent the complexity of items relating to other items and loading onto other dimensions. Network models, however, provide intuitive depictions of these interactions (Bringmann \& Eronen, 2018). Therefore, even though EFA loading matrices represent this complexity, it requires a certain level of psychometric expertise for a researcher to intuitively view the matrix this way. Moreover, network plots can reveal exactly which items are responsible for the cross-domain relationships in a way that an EFA loading matrix cannot.

\section{Loadings}

Recent simulation efforts, however, have demonstrated that network models can be used to estimate an EFA loading matrix equivalent. In a series of simulation studies, Hallquist, Wright, and Molenaar (2019) demonstrated that the network measure, node strength (i.e., the sum of a node's connections), was roughly redundant with CFA factor loadings. A notable finding in one of their studies was that a node's strength could potentially be a blend of connections within and between dimensions. Based on this result, they suggested that researchers should reduce the latent confounding of the network measure to avoid misrepresenting the relationships between components in the network.

Heeding this call, Christensen and Golino (under review) derived a measure called network loadings, which represents the standardization of node strength split between dimensions. More specifically, a node's strength was computed for only the connections it had to other nodes in each dimension of the network. They demonstrated that these network loadings could effectively estimate the simulated population (or true) loadings. Moreover, they found that network loadings more closely resembled EFA loadings, but also had some loadings of zero like CFA loadings. This suggests that the network loadings represent a complex structure that is between a saturated (EFA) and simple structure (CFA). In sum, they suggest that these network loadings can be used as an equivalent to factor loadings (for an example, see Table SI3 in the Supplementary Information).

Although these metrics are statistically redundant, they arguably differ in a substantive way. Factor loadings suggest that items "load" onto factors, which is provided by items being regressed on the factors. If interpreted in a substantive way, they represent how well one observable indicator is related to the factor - that is, how well an item represents or measures the latent factor. The substantive interpretation of

${ }^{5} \mathrm{~A}$ recent simulation study used the EGA approach and examined different community detection algorithms, finding that the Louvain (Blondel, Guillaume, Lambiotte, \& Lefebvre, 2008) and Walktrap algorithms were the most accurate and least biased of the eight algorithms tested (including two parallel analysis methods; Christensen, 2020). 
node strength does not suggest this, however, they may be epistemologically related. From a substantive standpoint, we argue that these network loadings represent each node's contribution to the emergence of a coherent dimension in the network. In this sense, we can connect the substantive meanings of network and factor loadings: the more one item contributes to a dimension's coherence, the more the item reflects the underlying dimension. A researcher's substantive interpretation will favor one interpretation over the other but ultimately they statistically resolve to roughly the same thing (Christensen \& Golino, under review; Guttman, 1953; Hallquist et al., 2019).

\section{Internal Structure Analysis}

\section{Internal Consistency}

Analyses that quantify the internal structure of questionnaires have been dominated by internal consistency measures, which are almost always measured with Cronbach's $\alpha$ (Cronbach, 1951; Flake et al., 2017; Hubley, Zhu, Sasaki, \& Gadermann, 2014). In a review of 50 validation studies randomly selected from Psychological Assessment and European Journal of Personality Assessment during the years 2011 and 2012, $\alpha$ was reported in $90 \%$ and $100 \%$ of the articles, respectively (Hubley et al., 2014). Similar numbers were obtained in a review of 35 studies in the Journal of Personality and Social Psychology during 2014, with 79\% of scales that included two or more items $(n=301)$ reporting $\alpha$ (Flake et al., 2017). More often than not, $\alpha$ was the sole measure of structural validation. In short, the use of $\alpha$ in validation is pervasive (McNeish, 2018).

Despite $\alpha$ 's prevalence, there are some serious issues (Dunn, Baguley, \& Brunsden, 2014; Sijtsma, 2009). These issues range from improper assumptions about the data (e.g., $\tau$-equivalent vs. congeneric models; Dunn et al., 2014; McNeish, 2018) to misconceptions about what it actually measures (Schmitt, 1996; Sijtsma, 2009). Although newer internal consistency measures (e.g., $\omega$; Dunn et al., 2014; McDonald, 1999; Zinbarg, Yovel, Revelle, \& McDonald, 2006) account for improper assumptions about the data, misconceptions about internal consistency still abound. One of the more persistent misconceptions is that internal consistency measures assess unidimensionality (Flake et al., 2017). This misconception likely stems from confusion over the difference between internal consistency (the extent to which items are interrelated) and homogeneity (a set of items that have a common cause; Green, Lissitz, \& Mulaik, 1977). Based on these definitions, internal consistency is necessary but not sufficient for homogeneity (Schmitt, 1996).

We believe that many misconceptions arise because there is a mismatch between what researchers intend to measure and what they are actually measuring. Much like validity, the psychometric concept of internal consistency seems divorced from how researchers think about it (Borsboom et al., 2004). This is because most researchers know that the items of their scale are interrelated - they were designed that way. When framed in this light, internal consistency measures are more of a "sanity check" than an informative measure. To better understand what researchers intend to measure, we can look at how they use these measures: Researchers use them to validate the consistency of the structure of their scales (Flake et al., 2017). That is, researchers use them to know whether their scale's structure is consistent which implies internal consistency and assumes homogeneity.

From a latent variable perspective, the solution is straightforward: test if a unidimensional model fits and compute an internal consistency measure (Flake et al., 2017; Green et al., 1977). From a psychometric network perspective, this is not the case. First, there is an inherent incompatibility with computing an internal consistency measure from the network perspective. Internal consistency measures are typically a variant on the ratio between the common covariance between items and the variance of those items (McNeish, 2018). In the estimation of networks, most of the common covariance is removed, leaving only the correlations between item-specific variance (Forbes, Wright, Markon, \& Krueger, 2017, 2019).

Second, scales and their items in networks are interrelated, usually with cross-connections occurring throughout. This is more than likely to be true for personality scales (Socan, 2000). Therefore, it's important to know whether a set of items are causally dependent and form a unidimensional network, but also whether they remain as a coherent subnetwork nested in the rest of the network. Said differently, questionnaires often contain scales that are assumed to be unidimensional but it's unclear whether these scales remain unidimensional when other items and scales are added (i.e., in a "multidimensional context"). Therefore, internal consistency measures do not capture whether scales (or dimensions) remain unidimensional within the context of other items and dimensions. Regardless of psychometric model, this seems to be a more informative measure of what most researchers want to know and say about their scalesthat they are unidimensional and internally consistent.

\section{Structural Consistency}

We refer to this measure as structural consistency, which we substantively define as the extent to which causally coupled components form a coherent sub-network within a network. Using extant terminology, structural consistency is the extent to which items in a dimension are homogeneous and interrelated given the multidimensional structure of the questionnaire. In other words, it is the combination of homogeneity and internal consistency in a multidimensional context. We view the inclusion of other dimensions as a particularly important conceptual feature because a dimension could have high homogeneity and internal consistency but when placed in the context of other related dimensions it's structure falls apart (i.e., it is no longer unidimensional). This renders the interpretation of that dimension in a multidimensional context relatively ambiguous even when its interpretation is clear in a unidimensional context (i.e., examined in isolation).

A recently developed approach called Bootstrap Exploratory Graph Analysis (bootEGA; Christensen \& Golino, 2019) can be used to estimate this measure. bootEGA applies a parametric and non-parametric bootstrap approach but for the structural consistency measure we focus on the parametric approach. The parametric approach begins by estimating a GLASSO network from the data and taking the inverse of the network to derive a covariance matrix. This covariance matrix is then used to simulate data with the 
same number of cases as the original data from a multivariate normal distribution.

EGA is then applied to this replicate data, obtaining each item's assigned dimension. This procedure is repeated until the desired number of samples is achieved (e.g., 500 samples). ${ }^{6}$ The result from this procedure is a sampling distribution for the total number of dimensions and each item's dimension allocation. Although a number of statistics can be computed, we focus on two: structural consistency and item stability. To derive both statistics, the original EGA results (i.e., empirically-derived dimensions) are used.

Structural consistency is derived by computing the proportion of times that each empirically-derived dimension is exactly (i.e., identical item composition) recovered from the replicate bootstrap samples. If a scale is unidimensional, then structural consistency reduces to the extent that the items in the scale form a single dimension-that is, the proportion of replicate samples that also return one dimension. The range that structural consistency can take is from 0 to 1. A dimension's structural consistency can only be 1 if the items in the a priori dimension conform to that dimension across all replicate samples. Such a measure leads to an important question: What's happening when a dimension is structurally inconsistent?

To answer this, item stability or the proportion of times that each item is identified in each empirically-derived dimension across the replicate samples can be computed. This relatively simple measure not only provides insight into which items may be causing structural inconsistency but also the other dimension(s) these items are being placed in. On the one hand, two items of our hypothetical dimension might be at the root of the structural inconsistency; on the other hand, it might be multiple items are at the root of the structural inconsistency. In either case, examining each item's replication proportions across dimensions can reveal whether they are forming a new separate dimension (only replicating in a new dimension), fit better with another dimension (replicating more with another dimension), or identify as multidimensional items (replicating equally across multiple dimensions). The latter two explanations can be verified using the network loading matrix. An example of these analyses are provided in the Structural Consistency section of the Supplementary Information.

In practice, the goal of structural consistency is to determine the extent in which a dimension is comprised of a set items that are homogeneous and interrelated in the context of other dimensions. The importance that a dimension of a questionnaire has a high structural consistency is up to the researcher's intent. For many dimensions in personality, items may be multidimensional, which will lead to lower values of structural consistency. Therefore, lower structural consistency is not a bad thing if it is what the researcher intends. More importantly, the items that are leading to the lower structural consistency can be identified with item stability statistics, which may help researchers decided whether an item is multidimensional or fits better with another dimension. At this point, it is too early to make recommendations for what "high" or "acceptable" structural consistency means. Ultimately, simulation studies are necessary to develop such standards.

\section{Discussion}

Questionnaires have been and will likely continue to be a standard format for the measurement of personality attributes. The validity of what questionnaires claim to measure, however, has rarely been explicated in the contemporary personality literature. Instead, psychometric models have been applied without much consideration of their causal implications. In this paper, we provided a review on the validity of personality trait questionnaires from the latent variable and psychometric network perspectives. The goal of our review was not to argue for one approach more than another but to elaborate on how questionnaires can be viewed as valid measures of personality traits or attributes. These views imply different substantive interpretations about the underlying data generating mechanisms, which are important for understanding the meaning of what's being measured and how psychometric measures substantively inform our measurement.

In our review, we took special interest in elaborating on the psychometric network perspective because few articles have focused on their measurement when applied to personality questionnaires. Much like latent variable models, psychometric network models have been readily applied by researchers without much consideration about their causal implications or how network measures should be interpreted in a psychological context (Bringmann et al., 2019). Based on our review, we propose a substantive interpretation of node strength (network loadings) that is appropriate in the context of dimensions and the overall network - that is, a node's contribution to the emergence of a coherent sub-network or network. This interpretation is by no means definite; however, we believe that it is a more appropriate interpretation than what has been put forward in the literature.

More specific to personality, we explicated an initial framework for how psychometric networks can be used to validate the structure of personality trait questionnaires. One point of emphasis was on reducing the redundancy of components in the network. This is because components of a network are defined as "unique" and "causally autonomous" (Cramer et al., 2012a). We described a novel approach to detect an item's redundancy with other items in the network, which can aid researchers in this endeavor. Moreover, we provided some general recommendations for removing or combining redundant items. Following from this emphasis, greater exploration of the unique components that represent the domain of personality traits is necessary so that a specific set of attributes can be defined. This is unlikely to be an easy task because personality traits are multifaceted and interrelated, which suggests that representation of a domain may be a matter of degree rather than clear cut definitions (Schmittmann et al., 2013; Schwaba et al., 2020).

This puts determining appropriate coverage of each trait's domain at the forefront of the psychometric network research agenda in personality. Indeed, determining appropriate coverage of each trait's domain is still an active area of research and requires more attention than it's been given in the past. In many cases, this will require sampling from attributes that may lie just outside of a

${ }^{6} 500$ replicate samples should be an adequate number to achieve an accurate estimate; however, researchers can increase this number to obtain greater precision. 
trait's domain. We recommend that researchers focus more on the extent to which attributes represent each domain rather than assuming an existing questionnaire's domain coverage is sufficient. One place to start is by examining the unique items of several personality questionnaires in a single network domain (e.g., Christensen et al., 2019). Multiple domains and outcome measures could also be included to help determine these boundaries (e.g., Afzali, Stewart, Seguin, \& Conrod, in press; Costantini, Richetin, et al., 2015; Schwaba et al., 2020).

When it comes to item and dimension analyses, many of the statistics for latent variable models (i.e., factor loadings and dimensions) are mathematically equivalent to psychometric network models (Christensen, 2020; Golino \& Epskamp, 2017; Hallquist et al., 2019). We argued that the key difference between these models is their substantive interpretations, which suggest very different data generating mechanisms (van Bork et al., 2019). At this point, disentangling these models is a nascent area of research.

Kan, van der Maas, and Levine (2019), for example, show how fit indices can be applied to network models so that they can be compared to confirmatory factor analysis models. They also demonstrated how the comparison of networks over groups could be achieved (see also Epskamp, 2019). van Bork et al. (2019) developed an approach to compare the likelihood that data was generated from a unidimensional factor model or sparse network model by assessing the proportion of partial correlations that have a different sign than the corresponding zero-order correlations and the proportion of partial correlations that are stronger than the corresponding zero order correlations (greater proportions for both increase the likelihood of the sparse network model). Approaches like these can be used to determine whether a latent variable or psychometric network model may be more appropriate for the data. Ultimately, we believe that the choice of model will not significantly affect the outcomes of these dimension-related analyses.

Finally, we introduced a novel measure, structural consistency, to quantify a questionnaire's internal structure. Part of the motivation for this measure was the need to move beyond measures of internal consistency, which we believe do not necessarily align with what researchers intend to measure. Notably, we do not view this measure as incompatible with internal consistency but rather complementary. As we discussed, a dimension could be homogeneous (i.e., unidimensional) and internally consistent (i.e., interrelated) but it may not remain homogeneous in a multidimensional context. Such a condition is likely to occur in personality measures where components of traits tend to be interrelated. In general, this measure adds to the internal structure methods that researchers can use for validating the structure of their questionnaire.

\section{Steps Toward External Validation}

To this point, we've described our conceptual framework for the structural validation of personality questionnaires from the network perspective. This framework leaves open questions related to external validation. How, for example, do outcome variables relate to the components in personality networks? What about covariates? How does this fit with contemporary trends for evaluating the unique predictive value of items? Moreover, what if the researcher is interested in relating the trait itself to outcomes rather than components? We briefly discuss these questions in turn.

Personality-outcome relations are a fundamental part of personality research and the validation of personality assessment instruments. These relations are just as fundamental to the network perspective as more traditional perspectives. Our suggestion for this is relatively simple: include the outcome(s) of interest in the network. Similarly, important covariates should also be added to the network. Indeed, Costantini, Richetin, et al. (2015) used this approach to evaluate how facets of conscientiousness were related to measures of self-control, working memory, selfreport behaviors related to conscientiousness, and implicit attitudes of conscientiousness descriptors. Similarly, Afzali et al. (in press) longitudinally examined items of the Substance Use Risk Profile Scale and their relations to cannabis and alcohol use in adolescences. These studies not only provide a more complex evaluation of the relations between personality and outcomes but also provide more targeted item generation for future measures (e.g., including more specific items measuring sensation-seeking personality indicators in substance use; Afzali et al., in press).

We propose that because networks are often estimated using the GLASSO approach, researchers can interpret the partial correlation coefficients between outcomes and components in the network as if they were entered into a regularized regression. Regularized regression has already been effectively used in the literature to evaluate personalityoutcome relations (Mõttus, Bates, Condon, Mroczek, \& Revelle, 2018; Seeboth \& Mõttus, 2018). To achieve a similar model, researchers could compute beta coefficients from the partial correlation coefficients for the outcome variable (see Haslbeck \& Waldorp, 2018). More directly, researchers could square these same partial correlation coefficients to derive partial $R^{2}$ values (or the residual variation explained by adding the variable to the network), which makes for more interpretable results (Haslbeck \& Waldorp, 2018).

Within our proposed framework, networks would be comprised of personality components rather than specific items or facets. On the surface, this seems to clash with recent articles (including this special issue) demonstrating the unique predictive value of items in personality-outcome associations. In our view, this conflict is relatively minimal; instead, we think that unique personality components should be tapping into the very same notion. Items should have unique predictive value if they have distinct causes beyond other items - just as personality components are conceptualized.

On the one hand, when considering the example of the items, "Hate being the center of attention," "Make myself the center of attention," "Like to attract attention," and "Dislike being the center of attention," there is unlikely to be unique predictive value of one item over another. On the other hand, unique items that do not have such an obvious overlap should remain as items and therefore unique components in the personality network. Therefore, we view personality components to be completely compatible 
with the unique predictive value of items while reducing homogeneous sets of items to their unique causes.

Finally, researchers may be interested in the relations between traits and outcomes. As mentioned in our section on validity, traits are viewed as a summary of the network's state. Based on this definition, a summary statistic could be computed and used to evaluate the relationship between traits and outcomes. Using network loadings, Christensen and Golino (under review) propose multiplying the loading matrix by the observed data to derive a weighted composite for each dimension (e.g., facets, traits) in a personality network. These composites could then be used in traditional analyses (e.g., zero-order correlation and regression) or as variables in a "higher-order" network with the outcome (and covariate) variables included. Following the same suggestions above, researchers could then square the outcome's partial correlation coefficients to derive the partial $R^{2}$ for the higher-order personality components in the network.

\section{Conclusion}

So, what are personality traits? At this point, it's clear that how researchers answer this question should affect the psychometric model they choose. In doing so, there are different considerations that should be made when developing and selecting items for their scales as well as how they should interpret the measures used to quantify their scales. In this article, we take the initial steps towards how researchers can go about this with psychometric network models. We by no means suggest that our views represent the views of all researchers using these models (including latent variable models); however, we have provided a foundation for future work and discussion. Undoubtedly, the successful application of psychometric network models in personality psychology requires explicit definition and formalization of their measurement (e.g., components), which we have provided (Costantini \& Perugini, 2012; Cramer et al., 2012b). We are optimistic that the continued development of measurement from a psychometric network perspective can move the theoretical and substantive assessment of personality traits forward.

\section{References}

Afzali, M. H., Stewart, S. H., Seguin, J. R., \& Conrod, P. (in press). The network constellation of personality and substance use: Evolution from early to late adolescence. European Journal of Personality. https: //doi.org/10.1002/per.2245

Allport, G. W. (1961). Pattern and growth in personality. Oxford, UK: Holt, Reinhart, \& Winston.

Ashton, M. C., \& Lee, K. (2005). A defence of the lexical approach to the study of personality structure. European Journal of Personality, 19, 5-24. https: //doi.org/10.1002/per.541

Baumert, A., Schmitt, M., Perugini, M., Johnson, W., Blum, G., Borkenau, P., ... Wrzus, C. (2017). Integrating personality structure, personality process, and personality development. European Journal of Personality, 31, 503-528. https://doi.org/10.1002/per. 2115

Blanken, T. F., Deserno, M. K., Dalege, J., Borsboom, D., Blanken, P., Kerkhof, G. A., \& Cramer, A. O. J. (2018). The role of stabilizing and communicating symptoms given overlapping communities in psychopathology networks. Scientific Reports, 8, 5854. https://doi.org/ 10.1038/s41598-018-24224-2

Blondel, V. D., Guillaume, J.-L., Lambiotte, R., \& Lefebvre, E. (2008). Fast unfolding of communities in large networks. Journal of Statistical Mechanics: Theory and Experiment, 2008, P10008. https://doi.org/10.1088/ 1742-5468/2008/10/P10008

Borkenau, P., \& Ostendorf, F. (1998). The Big Five as states: How useful is the five-factor model to describe intraindividual variations over time? Journal of Research in Personality, 32, 202-221. https://doi.org/10.1006/ jrpe.1997.2206

Borsboom, D. (2006). The attack of the psychometricians. Psychometrika, 71, 425-440. https://doi.org/10.1007/ s11336-006-1447-6

Borsboom, D. (2008). Psychometric perspectives on diagnostic systems. Journal of Clinical Psychology, 64, 1089-1108. https://doi.org/10.1002/jclp.20503

Borsboom, D. (2017). A network theory of mental disorders. World Psychiatry, 16, 5-13. https://doi.org/10.1002/ wps. 20375

Borsboom, D., Cramer, A. O. J., Kievit, R. A., Scholten, A. Z., \& Franic, S. (2009). The end of construct validity. In R. W. Lissitz (Ed.), The concept of validity: Revisions, new directions and applications (pp. 135-170). Charlotte, NC: IAP Information Age Publishing.

Borsboom, D., Cramer, A. O. J., Schmittmann, V. D., Epskamp, S., \& Waldorp, L. J. (2011). The small world of psychopathology. PLOS ONE, 6. https: //doi.org/10.1371/journal.pone.0027407

Borsboom, D., \& Mellenbergh, G. J. (2007). Test validity in cognitive assessment. In J. P. Leighton \& M. J. Gierl (Eds.), Cognitive diagnostic assessment for education: Theory and applications (pp. 85-116). New York, NY: Cambridge University Press. https://doi.org/10.1017/ cbo9780511611186.004\%20 
Borsboom, D., Mellenbergh, G. J., \& van Heerden, J. (2003). The theoretical status of latent variables. Psychological Review, 110, 203-219. https://doi.org/ 10.1037/0033-295X.110.2.203

Borsboom, D., Mellenbergh, G. J., \& van Heerden, J. (2004). The concept of validity. Psychological Review, 111, 1061-1071. https://doi.org/10.1037/0033-295X.111. 4.1061

Bringmann, L. F., Elmer, T., Epskamp, S., Krause, R. W., Schoch, D., Wichers, M., .. Snippe, E. (2019). What do centrality measures measure in psychology networks? Journal of Abnormal Psychology, 128, 892903. https://doi.org/10.1037/abn0000446

Bringmann, L. F., \& Eronen, M. I. (2018). Don't blame the model: Reconsidering the network approach to psychopathology. Psychological Review, 125, 606-615. https://doi.org/10.1037/rev0000108

Browne, M. W. (2001). An overview of analytic rotation in exploratory factor analysis. Multivariate Behavioral Research, 36, 111-150. https://doi.org/10.1207/ S15327906MBR3601_05

Cattell, R. B. (1946). Description and measurement of personality. New York, NY: World Book Company.

Cervone, D. (2005). Personality architecture: Withinperson structures and processes. Annual Review of Psychology, 56, 423-452. https://doi.org/10.1146/ annurev.psych.56.091103.070133

Christensen, A. P. (2020). Towards a network psychometrics approach to assessment: Simulations for redundancy, dimensionality, and loadings (Unpublished doctoral dissertation). University of North Carolina at Greensboro, Greensboro, NC, USA. https://doi.org/10. 31234/osf.io/84kgd

Christensen, A. P., Cotter, K. N., \& Silvia, P. J. (2019). Reopening openness to experience: A network analysis of four openness to experience inventories. Journal of Personality Assessment, 101, 574-588. https://doi.org/ 10.1080/00223891.2018.1467428

Christensen, A. P., \& Golino, H. (2019). Estimating the stability of the number of factors via Bootstrap Exploratory Graph Analysis: A tutorial. PsyArXiv. https: //doi.org/10.31234/osf.io/9deay

Christensen, A. P., \& Golino, H. (under review). Statistical equivalency of factor and network loadings. PsyArXiv. https://doi.org/10.31234/osf.io/xakez

Condon, D. M. (2018). The SAPA personality inventory: An empirically-derived, hierarchically-organized selfreport personality assessment model. PsyArXiv. https: //doi.org/10.31234/osf.io/sc4p9

Connelly, B. S., Ones, D. S., Davies, S. E., \& Birkland, A. (2014). Opening up openness: A theoretical sort following critical incidents methodology and a metaanalytic investigation of the trait family measures. Journal of Personality Assessment, 96, 17-28. https: //doi.org/10.1080/00223891.2013.809355

Costantini, G., Epskamp, S., Borsboom, D., Perugini, M., Mõttus, R., Waldorp, L. J., \& Cramer, A. O. J. (2015). State of the aRt personality research: A tutorial on network analysis of personality data in R. Journal of Research in Personality, 54, 13-29. https://doi.org/10. 1016/j.jrp.2014.07.003

Costantini, G., \& Perugini, M. (2012). The definition of components and the use of formal indexes are key steps for a successful application of network analysis in personality psychology. European Journal of Personality, 26, 434-435. https://doi.org/10.1002/ per.1869

Costantini, G., Richetin, J., Borsboom, D., Fried, E. I., Rhemtulla, M., \& Perugini, M. (2015). Development of indirect measures of conscientiousness: Combining a facets approach and network analysis. European Journal of Personality, 29, 548-567. https://doi.org/ 10.1002/per.2014

Costantini, G., Richetin, J., Preti, E., Casini, E., Epskamp, S., \& Perugini, M. (2019). Stability and variability of personality networks. A tutorial on recent developments in network psychometrics. Personality and Individual Differences, 136, 68-78. https://doi.org/ 10.1016/j.paid.2017.06.011

Cramer, A. O. J. (2012). Why the item " $23+1$ " is not in a depression questionnaire: Validity from a network perspective. Measurement: Interdisciplinary Research \& Perspective, 10, 50-54. https://doi.org/10.1080/ 15366367.2012.681973

Cramer, A. O. J., van der Sluis, S., Noordhof, A., Wichers, M., Geschwind, N., Aggen, S. H., ... Borsboom, D. (2012a). Dimensions of normal personality as networks in search of equilibrium: You can't like parties if you don't like people. European Journal of Personality, 26, 414-431. https://doi.org/10.1002/per.1866

Cramer, A. O. J., van der Sluis, S., Noordhof, A., Wichers, M., Geschwind, N., Aggen, S. H., ... Borsboom, D. (2012b). Measurable like temperature or mereological like flocking? On the nature of personality traits. European Journal of Personality, 26, 451-459. https: //doi.org/10.1002/per.1879

Cramer, A. O. J., Waldrop, L. J., van der Maas, H. L., \& Borsboom, D. (2010). Comorbidity: A network perspective. Behavioral and Brain Sciences, 33, 137150. https://doi.org/10.1017/S0140525X09991567

Cronbach, L. J. (1951). Coefficient alpha and the internal structure of tests. Psychometrika, 16, 297-334. https: //doi.org/10.1007/BF02310555

Cronbach, L. J., \& Meehl, P. E. (1955). Construct validity in psychological tests. Psychological Bulletin, 52, 281302. https://doi.org/10.1037/h0040957

Delignette-Muller, M. L., \& Dutang, C. (2015). fitdistrplus: An $\mathrm{R}$ package for fitting distributions. Journal of Statistical Software, 64, 1-34. https://doi.org/10.18637/ jss.v064.i04

DeVellis, R. F. (2017). Scale development: Theory and applications (4th ed.). Thousand Oaks, CA: SAGE Publications.

DeYoung, C. G., Quilty, L. C., \& Peterson, J. B. (2007). Between facets and domains: 10 aspects of the Big Five. Journal of Personality and Social Psychology, 93, 880896. https://doi.org/10.1037/0022-3514.93.5.880 
Dinic, B. M., Wertag, A., Tomasevic, A., \& Sokolovska, V. (2020). Centrality and redundancy of the Dark Tetrad traits. Personality and Individual Differences, 155, 109621. https://doi.org/10.1016/j.paid.2019.109621

Dunn, T. J., Baguley, T., \& Brunsden, V. (2014). From alpha to omega: A practical solution to the pervasive problem of internal consistency estimation. British Journal of Psychology, 105, 399-412. https://doi.org/10.1111/ bjop.12046

Epskamp, S. (2019). psychonetrics: Structural equation modeling and confirmatory network analysis. $\mathrm{R}$ package verson 0.3.3. Retrieved from https://CRAN. R-project.org/package $=$ psychonetrics

Epskamp, S., \& Fried, E. I. (2018). A tutorial on regularized partial correlation networks. Psychological Methods, 23, 617-634. https://doi.org/10.1037/met0000167

Epskamp, S., Maris, G., Waldrop, L. J., \& Borsboom, D. (2018a). Network psychometrics. In P. Irwing, D. Hughes, \& T. Booth (Eds.), The Wiley handbook of psychometric testing, 2 volume set: A multidisciplinary reference on survey, scale and test development. New York, NY: Wiley. https://doi.org/10.1002/ 9781118489772.ch30

Epskamp, S., Waldorp, L. J., Mõttus, R., \& Borsboom, D. (2018b). The Gaussian Graphical Model in crosssectional and time-series data. Multivariate Behavioral Research, 4, 1-28. https://doi.org/10.1080/00273171. 2018.1454823

Flake, J. K., Pek, J., \& Hehman, E. (2017). Construct validation in social and personality research: Current practice and recommendations. Social Psychological and Personality Science, 8, 370-378. https://doi.org/ $10.1177 / 1948550617693063$

Fleeson, W. (2001). Toward a structure- and processintegrated view of personality: Traits as density distributions of states. Journal of Personality and Social Psychology, 80, 1011-1027. https://doi.org/10.1037/ 0022-3514.80.6.1011

Fleeson, W., \& Gallagher, P. (2009). The implications of Big Five standing for the distribution of trait manifestation in behavior: Fifteen experience-sampling studies and a meta-analysis. Journal of Personality and Social Psychology, 97, 1097-1114. https://doi.org/10.1037/ a0016786

Fleeson, W., \& Jayawickreme, E. (2015). Whole trait theory. Journal of Research in Personality, 56, 82-92. https://doi.org/10.1016/j.jrp.2014.10.009

Forbes, M. K., Wright, A. G. C., Markon, K. E., \& Krueger, R. F. (2017). Evidence that psychopathology symptom networks have limited replicability. Journal of Abnormal Psychology, 126, 969-988. https://doi.org/10.1037/ abn0000276

Forbes, M. K., Wright, A. G., Markon, K. E., \& Krueger, R. F. (2019). Quantifying the reliability and replicability of psychopathology network characteristics. Multivariate Behavioral Research. https: //doi.org/10.1080/00273171.2019.1616526
Fortunato, S. (2010). Community detection in graphs. Physics Reports, 486, 75-174. https://doi.org/10.1016/ j.physrep.2009.11.002

Friedman, J., Hastie, T., \& Tibshirani, R. (2008). Sparse inverse covariance estimation with the graphical lasso. Biostatistics, 9, 432-441. https://doi.org/10.1093/ biostatistics/kxm045

Goldberg, L. R. (1993). The structure of phenotypic personality traits. American Psychologist, 48, 26-34. https://doi.org/10.1037/0003-066X.48.1.26

Golino, H., \& Christensen, A. P. (2020). EGAnet: Exploratory Graph Analysis: A framework for estimating the number of dimensions in multivariate data using network psychometrics. Retrieved from https://CRAN.R-project. org/package $=$ EGAnet

Golino, H., \& Demetriou, A. (2017). Estimating the dimensionality of intelligence like data using Exploratory Graph Analysis. Intelligence, 62, 54-70. https://doi.org/10.1016/j.intell.2017.02.007

Golino, H., \& Epskamp, S. (2017). Exploratory Graph Analysis: A new approach for estimating the number of dimensions in psychological research. PloS ONE, 12, e0174035. https://doi.org/10.1371/journal.pone. 0174035

Golino, H., Shi, D., Christensen, A. P., Garrido, L. E., Nieto, M. D., Sadana, R., ... Martinez-Molina, A. (in press). Investigating the performance of Exploratory Graph Analysis and traditional techniques to identify the number of latent factors: A simulation and tutorial. Psychological Methods. https://doi.org/10. 1037/met0000255

Green, S. B., Lissitz, R. W., \& Mulaik, S. A. (1977). Limitations of coefficient alpha as an index of test unidimensionality. Educational and Psychological Measurement, 37, 827-838. https://doi.org/10.1177/ 001316447703700403

Guttman, L. (1953). Image theory for the structure of quantitative variates. Psychometrika, 18, 277-296.

Hallquist, M., Wright, A. C. G., \& Molenaar, P. C. M. (2019). Problems with centrality measures in psychopathology symptom networks: Why network psychometrics cannot escape psychometric theory. Multivariate Behavioral Research. https://doi.org/10. 1080/00273171.2019.1640103

Hamaker, E. L., Nesselroade, J. R., \& Molenaar, P. C. M. (2007). The integrated trait-state model. Journal of Research in Personality, 41, 295-315. https://doi.org/ 10.1016/j.jrp.2006.04.003

Haslbeck, J. M. B., \& Waldorp, L. J. (2015). mgm: Structure estimation for time-varying mixed graphical models in high-dimensional data. arXiv. Retrieved from https: //arxiv.org/abs/1510.06871

Haslbeck, J. M. B., \& Waldorp, L. J. (2018). How well do network models predict observations? On the importance of predictability in network models. Behavior Research Methods, 50, 853-861. https://doi. org/10.3758/s13428-017-0910-x 
Hogan, R., \& Foster, J. (2016). Rethinking personality. International Journal of Personality Psychology, 2, 3743. Retrieved from http://ijpp.rug.nl/article/view/25245

Hubley, A. M., Zhu, S. M., Sasaki, A., \& Gadermann, A. M. (2014). Synthesis of validation practices in two assessment journals: Psychological Assessment and the European Journal of Psychological Assessment. In B. Zumbo \& E. Chan (Eds.), Validity and validation in social, behavioral, and health sciences (pp. 193-213). Cham, CH: Springer.

John, O. P., \& Srivastava, S. (1999). The Big Five trait taxonomy: History, measurement, and theoretical perspectives. In L. A. Pervin \& O. P. John (Eds.), Handbook of personality: Theory and research (2nd ed., pp. 159-181). New York, NY: Guilford Press.

Kan, K.-J., van der Maas, H. L. J., \& Levine, S. Z. (2019). Extending psychometric network analysis: Empirical evidence against $g$ in favor of mutualism? Intelligence, 73, 52-62. https://doi.org/10.1016/j.intell.2018.12. 004

Kane, M. T. (2013). Validating the interpretations and uses of test scores. Journal of Educational Measurement, 50, 1-73. https://doi.org/10.1111/jedm.12000

Kelley, T. L. (1927). Interpretation of educational measurements. New York, NY: MacMillan.

Kruis, J., \& Maris, G. (2016). Three representations of the Ising model. Scientific Reports, 6, srep34175. https://doi.org/10.1038/srep34175

Lauritzen, S. L. (1996). Graphical models. Oxford, UK: Clarendon Press.

Lee, K., \& Ashton, M. C. (2004). Psychometric properties of the HEXACO personality inventory. Multivariate Behavioral Research, 39, 329-358. https://doi.org/10. 1207/s15327906mbr3902_8

Lee, K., \& Ashton, M. C. (2018). Psychometric properties of the HEXACO-100. Assessment, 25, 543-556. https: //doi.org/10.1177/1073191116659134

Loevinger, J. (1957). Objective tests as instruments of psychological theory. Psychological Reports, 3, 635694.

Markus, K. A., \& Borsboom, D. (2013). Frontiers of test validity theory: Measurement, causation, and meaning. New York, NY: Routledge. https://doi.org/10.4324/ 9780203501207

Marsman, M., Borsboom, D., Kruis, J., Epskamp, S., van Bork, R., Waldorp, L. J., ... Maris, G. (2018). An introduction to network psychometrics: Relating Ising network models to item response theory models. Multivariate Behavioral Research, 53, 15-35. https: //doi.org/10.1080/00273171.2017.1379379

McCrae, R. R. (2015). A more nuanced view of reliability: Specificity in the trait hierarchy. Personality and Social Psychology Review, 19, 97-112. https://doi.org/10. 1177/1088868314541857

McCrae, R. R., \& Costa, P. T. (1987). Validation of the five-factor model of personality across instruments and observers. Journal of Personality and Social Psychology,
52, 81-90. https://doi.org/10.1037/0022-3514.52.1. 81

McCrae, R. R., \& Costa, P. T. (2008). The five-factor theory of personality. In O. P. John, R. W. Robins, \& L. A. Pervin (Eds.), Handbook of personality: Theory and research (3rd ed., pp. 159-181). New York, NY: Guilford Press.

McCrae, R. R., Costa, P. T., Ostendorf, F., Angleitner, A., Hreb̌ckova, M., Avia, M. D., ... Smith, P. B. (2000). Nature over nurture: Temperament, personality, and life span development. Journal of Personality and Social Psychology, 78, 173-186. https://doi.org/10. 1037//0022-3514.7S.1.17

McCrae, R. R., \& Mõttus, R. (2019). A new psychometrics: What personality scales measure, with implications for theory and assessment. Current Directions in Psychological Science.

McDonald, R. P. (1999). Test theory: A unified treatment. New York, NY: Taylor \& Francis. https://doi.org/10. 4324/9781410601087

McDonald, R. P. (2003). Behavior domains in theory and in practice. Alberta Journal of Educational Research, 49, 212-230.

McNeish, D. (2018). Thanks coefficient alpha, we'll take it from here. Psychological Methods, 23, 412-433. https://doi.org/10.1037/met0000144

Messick, S. (1989). Meaning and values in test validation: The science and ethics of assessment. Educational Researcher, 18, 5-11. https://doi.org/10. 3102/0013189X018002005

Messick, S. (1995). Standards of validity and the validity of standards in performance asessment. Educational Measurement: Issues and Practice, 14, 5-8. https: //doi.org/10.1111/j.1745-3992.1995.tb00881.x

Mõttus, R., \& Allerhand, M. (2017). Why do traits come together? The underlying trait and network approaches. In V. Ziegler-Hill \& T. K. Shackelford (Eds.), SAGE handbook of personality and individual differences: The science of personality and individual differences (pp. 122). London, UK: SAGE Publications.

Mõttus, R., Bates, T., Condon, D. M., Mroczek, D., \& Revelle, W. (2018). Leveraging a more nuanced view of personality: Narrow characteristics predict and explain variance in life outcomes. PsyArXiv. https://doi.org/10. 31234/osf.io/4q9gv

Nowick, K., Gernat, T., Almaas, E., \& Stubbs, L. (2009). Differences in human and chimpanzee gene expression patterns define an evolving network of transcription factors in brain. Proceedings of the National Academy of Sciences, 106, 22358-22363. https://doi.org/10.1073/ pnas.0911376106

Perugini, M., Costantini, G., Hughes, S., \& De Houwer, J. (2016). A functional perspective on personality. International Journal of Psychology, 51, 33-39. https: //doi.org/10.1002/ijop.12175

Pervin, L. A. (1994). A critical analysis of current trait theory. Psychological Inquiry, 5, 103-113. https: //doi.org/10.1207/s15327965pli0502_1 
Perez, M.-E., \& Pericchi, L. R. (2014). Changing statistical significance with the amount of information: The adaptive $\alpha$ significance level. Statistics \& Probability Letters, 85, 20-24. https://doi.org/10.1016/j.spl.2013. 10.018

Pons, P., \& Latapy, M. (2006). Computing communities in large networks using random walks. Journal of Graph Algorithms and Applications, 10, 191-218. https: //doi.org/10.7155/jgaa.00185

Rauthmann, J. F., Horstmann, K. T., \& Sherman, R. A. (2019). Do self-reported traits and aggregated states capture the same thing? A nomological perspective on trait-state homomorphy. Social Psychological and Personality Science, 10, 596-611. https://doi.org/10. 1177/1948550618774772

Rauthmann, J. F., \& Sherman, R. A. (2018). The description of situations: Towards replicable domains of psychological situation characteristics. Journal of Personality and Social Psychology, 482-488. https: //doi.org/10.1037/pspp0000162

R Core Team. (2020). R: A language and environment for statistical computing. Vienna, Austria: R Foundation for Statistical Computing. Retrieved from https://www. R-project.org/

Read, S. J., Monroe, B. M., Brownstein, A. L., Yang, Y., Chopra, G., \& Miller, L. C. (2010). A neural network model of the structure and dynamics of human personality. Psychological Review, 117, 61-92. https: //doi.org/10.1037/a0018131

Revelle, W. (2019). psychTools: Tools to accompany the 'psych' package for psychological research. Evanston, Illinois: Northwestern University. Retrieved from https://CRAN.R-project.org/package $=$ psychTools

Sass, D. A., \& Schmitt, T. A. (2010). A comparative investigation of rotation criteria within exploratory factor analysis. Multivariate Behavioral Research, 45, 73-103. https://doi.org/10.1080/ 00273170903504810

Schmitt, N. (1996). Uses and abuses of coefficient alpha. Psychological Assessment, 8, 350-353. https://doi.org/ 10.1037/1040-3590.8.4.350

Schmittmann, V. D., Cramer, A. O. J., Waldorp, L. J., Epskamp, S., Kievit, R. A., \& Borsboom, D. (2013). Deconstructing the construct: A network perspective on psychological phenomena. New Ideas in Psychology, 31, 43-53. https://doi.org/10.1016/j.newideapsych. 2011.02.007

Schwaba, T., Rhemtulla, M., Hopwood, C. J., \& Bleidorn, W. (2020). The facet atlas: Using network analysis to describe the blends, cores, and peripheries of personality structure. PsyArXiv. https://doi.org/10. 31234/osf.io/zskfu

Seeboth, A., \& Mõttus, R. (2018). Successful explanations start with accurate descriptions: Questionnaire items as personality markers for more accurate predictions. European Journal of Personality, 32, 186-201. https: //doi.org/10.1002/per.2147

Sijtsma, K. (2009). On the use, the misuse, and the very limited usefulness of Cronbach's alpha.
Psychometrika, 74, 107-120. https://doi.org/10.1007/ S11336-008-9101-0

Socan, G. (2000). Assessment of reliability when test items are not essentially $\tau$-equivalent. Developments in Survey Methodology, 15, 23-35.

van Bork, R., Rhemtulla, M., Waldorp, L. J., Kruis, J., Rezvanifar, S., \& Borsboom, D. (2019). Latent variable models and networks: Statistical equivalence and testability. Multivariate Behavioral Research, 1-24. https://doi.org/10.1080/00273171.2019.1672515

van der Maas, H. L. J., Dolan, C. V., Grasman, R. P. P. P., Wicherts, J. M., Huizenga, H. M., \& Raijmakers, M. E. J. (2006). A dynamical model of general intelligence: The positive manifold of intelligence by mutualism. Psychological Review, 113, 842-861. https://doi.org/ 10.1037/0033-295X.113.4.842

Wood, D., Gardner, M. H., \& Harms, P. D. (2015). How functionalist and process approaches to behavior can explain trait covariation. Psychological Review, 122, 84-111. https://doi.org/10.1037/a0038423

Zhang, B., \& Horvath, S. (2005). A general framework for weighted gene co-expression network analysis. Statistical Applications in Genetics and Molecular Biology, 4, 17. https://doi.org/10.2202/1544-6115. 1128

Zinbarg, R. E., Yovel, I., Revelle, W., \& McDonald, R. P. (2006). Estimating generalizability to a latent variable common to all of a scale's indicators: A comparison of estimators for $\omega \mathrm{h}$. Applied Psychological Measurement, 30, 121-144. https://doi.org/10.1177/ 0146621605278814 


\section{Supplementary Information}

\section{Validation Example Using the SAPA Inventory}

To begin our example of structural validation from the psychometric network perspecitve, several pacakges need to be installed. From these packages, the psychTools (Revelle, 2019) and EGAnet (Golino \& Christensen, 2020) pacakges must be loaded.

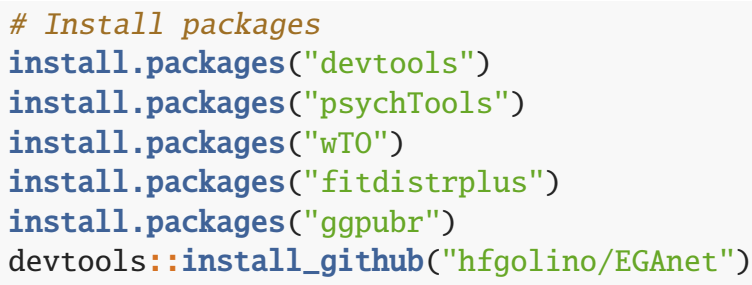

The psychTools package contains the SAPA inventory data that will be used for the example. More details about the sample can be found using the code ?spi or in Condon's (2018) SAPA inventory manual. Prior to any analyses, the items that correspond to the five-factor model (FFM; McCrae \& Costa, 1987) should be obtained with the following code:

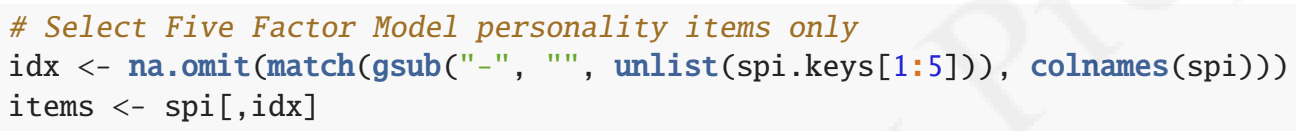

Node redundancy

These items can now be used in the node.redundant function available in the EGAnet pacakge. For the purposes of our example, we will use the weighted topological overlap approach-applied using the wTO package (Gysi, Voigt, de Miranda Fragoso, Almaas, \& Nowick, 2018) - and adaptive alpha (Perez \& Pericchi, 2014) multiple comparisons correction to determine which items are redundant.

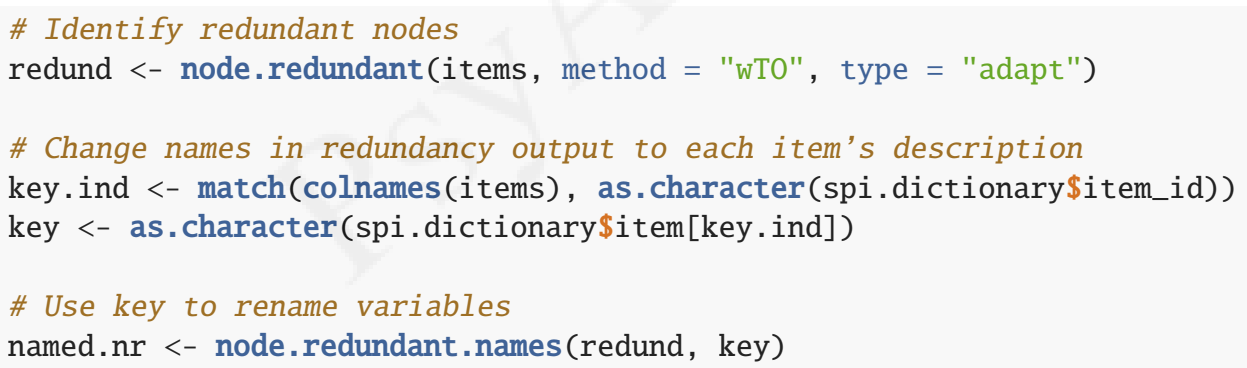

The output of the node. redundant function will correspond to the column names of the items in our data. The SAPA data is labeled with ambiguous names (e.g., q_565), which will need to be converted to item descriptions (e.g., Dislike being the center of attention.) to be used in the next step. The node.redundant.names function will accept a key that maps the ambiguous column names to the item descriptions. The input for the argument key should be a vector with item descriptions that correspond to the order of the column names.

The output from both functions will be a list, \$redundant, containing lists of redundant items. Each list nested in the \$redundant list will be named after an item that is redundant with other items. The \$redundant list is structured so that items with the greatest number of redundant items are placed at the top. An example of what one of these item lists look like is presented below (Table SI1).

The name of this item list, 'Am full of ideas. ', is the item that is redundant with the items listed below it. The $\$ r e d u n d a n t$ list is structured this way for each item that is redundant with other items. This structure, however, is verbose and difficult to manage. In order to navigate the process of merging items, the function node.redundant. combine should be used. 
Table SI1. Example of first element in redundancy list

Am full of ideas.

Am able to come up with new and different ideas.

Am an original thinker.

Love to think up new ways of doing things.

Have a vivid imagination.

\# Combining redundant responses

combined.nr <- node.redundant.combine(named.nr, type = "latent")

When entering this code, the R console will output each item list with a selection of possible redundant nodes (see Figure SI1) and an associated "redundancy chain" plot will appear (see Figure SI2). When examining the possible redundant nodes, the reader may notice that there were four items identified previously-that is, 'Am able to come up with new and different ideas.', 'Am an original thinker.', 'Love to think up new ways of doing things.', and 'Have a vivid imagination.' (Table SI1).

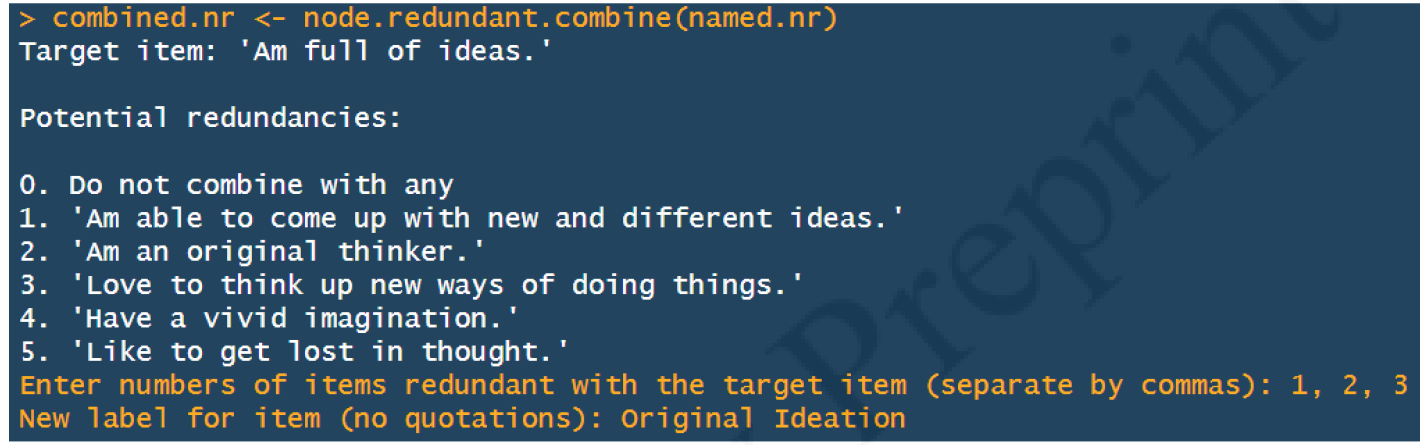

Figure SI1. An example of the menu that appears for each redundant item

The fifth option in Figure SI1, 'Like to get lost in thought.' ', was not redundant with the target item. It was, however, redundant with another item in our potential responses (i.e., 'Have a vivid imagination. '). Some items that are redundant with the original item may also be redundant with other items that are not redundant with the original item, forming a so-called "redunancy chain." The redundancy chain plot depicts this chain, which can be useful for deciding how redundant nodes should be combined by informing researchers about the overlap of near nodes. In these plots (see Figure $\mathrm{SI} 2$ ), the connections between items represent redundancies that have been determined to be statistically significant and the thickness of the connections correspond to those items' connections in the network (i.e., regularized partial correlations). 


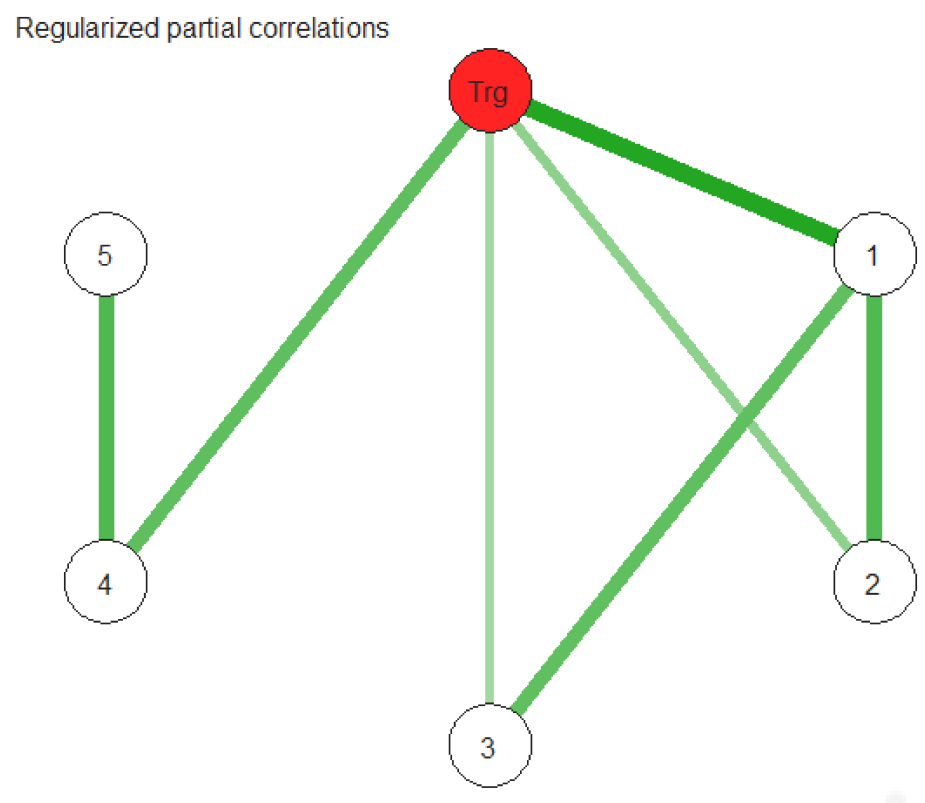

Figure SI2. An example of a redundancy chain plot. The red node indicates the target item and the white nodes with numbers correspond to the numbered options (Figure SI1). A connection represents significant overlap determined by the redundancy analysis and the thickness of the connection represents the regularized partial correlation between the nodes in the network.

From the redundancy chain plot (Figure SI2), the fifth item is shown to be redundant with the fourth item but not the target item (shown in red; Figure SI2). The target item should be the focus when considering which items are redundant. This can be determined by examining which items' content are redundant with the content of the target item. The redundancy chain plot can be consulted to determine whether multiple items are redundant with the target item. When consulting the redundancy chain plot, researchers should pay particular attention to cliques - a fully connected set of nodes. In Figure SI2, there are two 3-cliques (or triangles) with the target item (i.e., $\operatorname{Trg}-1-2$ and $\operatorname{Trg}-1-3$ ).

In a psychometric network, these triangles contribute to a measure known as the clustering coefficient or the extent to which a node's neighbors are connected to each other. Based on this statistical definition, the clustering coefficient has recently been considered as a measure of redundancy in networks (Costantini et al., 2019; Dinic, Wertag, Tomasevic, \& Sokolovska, 2020). In this same sense, these triangles suggest that these items are likely to have particularly high overlap. Therefore, triangles in these redundancy chain plots can be used as a heuristic for selecting items. Indeed, when inspecting these items, they appear to be relatively redundant with one another (Figure SI1).

In our example, we selected these items by inputing their numbers into the R console with commas separating them (i.e., $1,2,3)$. If the researcher decides that all items are unique with respect to the target item, then they can type $\mathbb{0}$, which will not combine any items and move to the next target item. If the user selects items, then they will be prompted to label the new component, which we labeled Original Ideation (Figure SI1).

The argument type will choose how to handle forming a unique component (i.e., latent variable or sum scores). We've chosen "latent", which will compute a unidimensional reflective latent variable. The function will remove the selected items from the data and replace them with the new component score. This completes the first target item. 


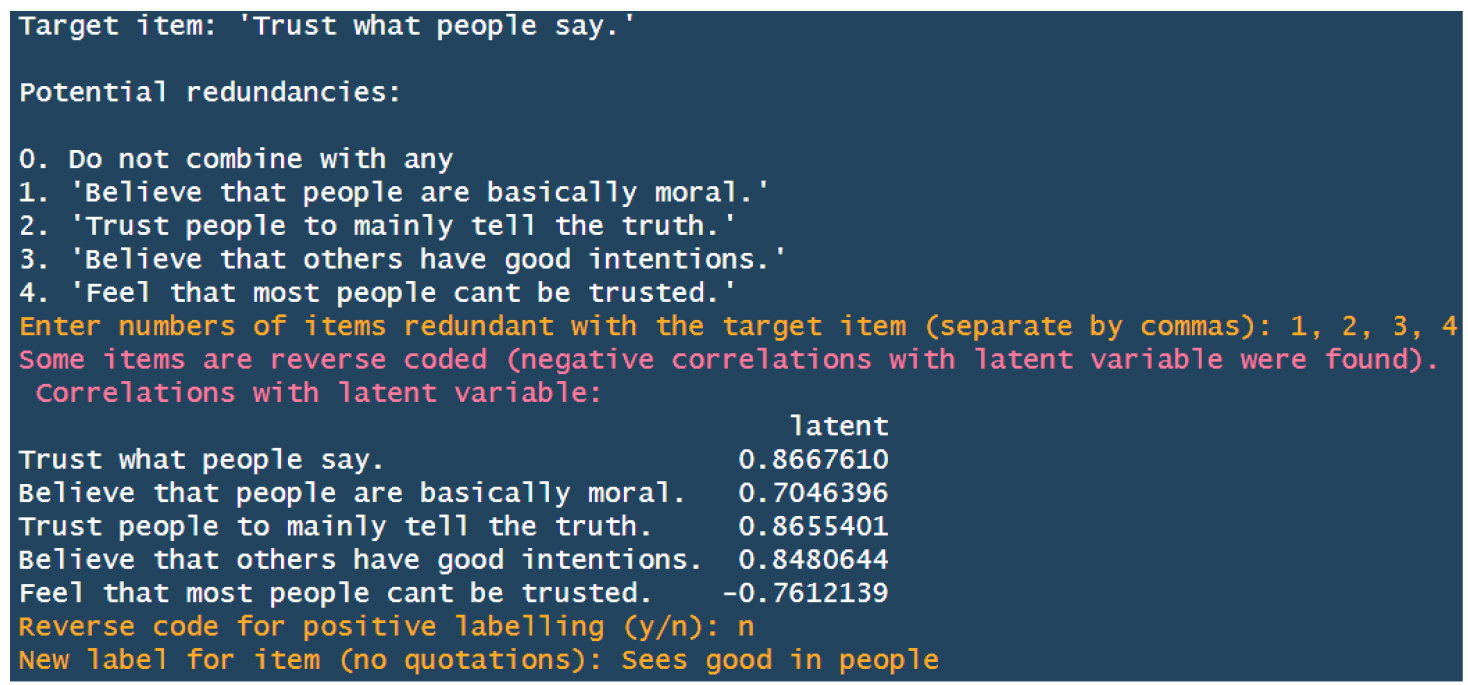

Figure SI3. An example of items that are reverse keyed and the prompt used to manage labeling.

For the second target item (Figure SI3), 'Trust what people say. ', we combined it with the other possible redundant items. Notably, there was one item that was reverse keyed, 'Feel that most people cant be trusted', which was negatively correlated with the latent variable. Because there was an item negatively correlated with the latent variable, a secondary prompt appears asking to reverse key the latent variable so that the label can go in the researcher's desired direction. In review of the correlations, we can see that the latent variable is positively keyed already; therefore, we entered ' $n$ ' and labeled the component. If, however, the signs of the correlations were the inverse, then the user could enter ' $y$ ', which would reverse the meaning of the latent variable towards a postively keyed orientation. The function will proceed through the rest of the redundant items until all have been handled.

Upon completing this process, the node.redundant. combine function will output a new data matrix (\$data) and a matrix containing items that were selected to be redundant with one another (\$merged). The new data will have the column names specified in the combination process with values representing either latent variable or sum scores for the combined items (i.e., components). Items that were not considered to be redundant with other items will be returned with their original values. The output of \$merged is useful for documenting what was done, making the choices in the process transparent. We have included a .csv file containing our output \$merged in our supplementary information for readers to review, assess, and follow along with this example.

\section{Dimensionality}

After the combination process is complete, exploratory graph analysis (EGA; Golino \& Epskamp, 2017; Golino et al., in press) can be applied. The following code can be used:

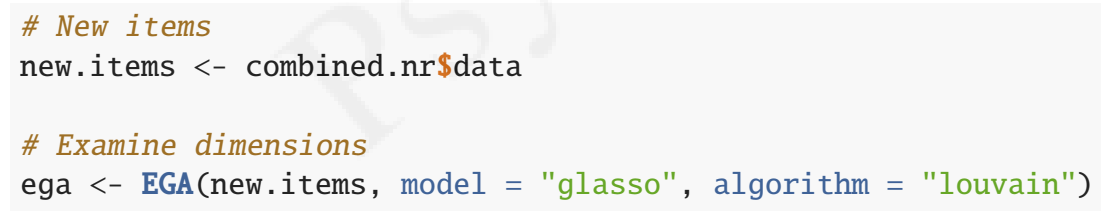




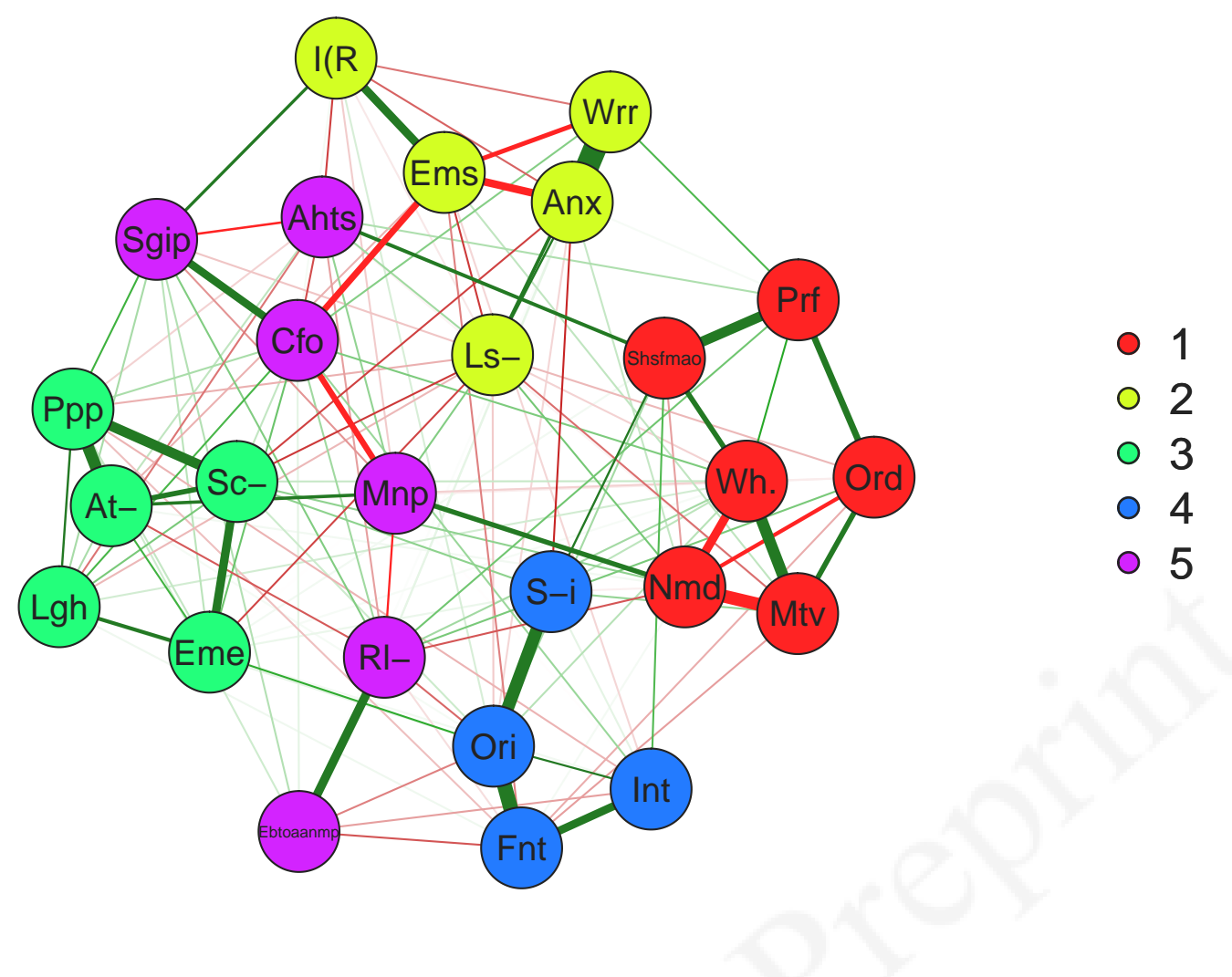

Figure S14. Depiction of the dimensions identified using exploratory graph analysis. The color of the nodes represent the dimensions and the thickness of the lines represent the magnitude of the partial correlations (green = positive; red $=$ negative).

EGA has been recently adapted to include the Louvain community detection algorithm (Blondel, Guillaume, Lambiotte, \& Lefebvre, 2008), which has demonstrated comparable or better performance than the Walktrap algorithm (Pons \& Latapy, 2006) in identifying dimensions (Christensen, 2020). For this reason, we estimated the dimensions of the network using the Louvain algorithm. Based on Figure SI4, there are five dimensions identified for these components. To view the composition of these dimensions, the following code should be entered:

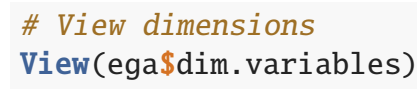

When reviewing the component content of these dimensions (Table SI2), these factors directly correspond to the FFM. Dimensions 1, 2, 3, 4 and 5 reflect conscientiousness, neuroticism, extraversion, openness to experience, and agreeableness, respectively. Although these components were empirically comprised of FFM items, this finding is an empirical validation of the effectiveness of the redundancy and dimensionality analyses. Next, we can investigate each node's contribution to the coherence of these dimensions using the net. loads function.

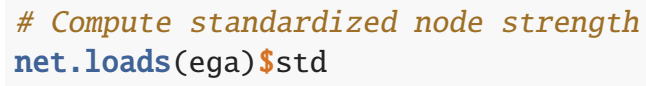

The net. loads function computes each node's standardized node strength for each dimension in the network. Therefore, Table SI3 can be interpreted similarly as an exploratory factor analysis loading matrix. When examining the network loadings matrix, there were a couple things worth noting. First, the network loadings are much smaller than the loadings of a traditional factor loading matrix. The largest loading is 0.409 for the original ideation component in the openness to experience dimension. By traditional factor analysis standards, this is a weak factor loading. This difference in the magnitude of the loadings is due to the association measure underlying the computation of the loading - that is, partial vs. zero-order correlations. The network loadings thus represent partial correlation loadings, meaning that 0.409 is actually a very large loading.

A key point moving forward will be to establish norms for what constitutes a small, moderate, and large network loading. It seems fair to suggest that effect sizes for multiple regression may hold for network loadings; however, the $f^{2}$ metric is likely to be more confusing for practical researchers than not (Cohen, 1992). Instead, using effect sizes that typically translate from these $f^{2}$ might be more interpretable, specifically effect sizes of $.10, .30$, and .50 corresponding to small, moderate, 
Table SI2. Item identification in EGA dimensions

\begin{tabular}{lc}
\hline Items & Dimension \\
\hline Work hard. & 1 \\
Neglect my duties. & 1 \\
Perfectionist & 1 \\
Orderly & 1 \\
Motivated & 1 \\
Set high standards for myself and others. & 1 \\
Worrier & 2 \\
Anxious & 2 \\
Low self-esteem & 2 \\
Irritable (R) & 2 \\
Emotional stability & 2 \\
People person & 3 \\
Attention-seeking & 3 \\
Social-efficacy & 3 \\
Laugher & 3 \\
Express myself easily. & 3 \\
Original ideation & 4 \\
Fantasy & 4 \\
Introspective & 4 \\
Self-assessed intelligence & 4 \\
Concerned for others & 4 \\
Manipulative & 5 \\
Sees good in people & 5 \\
Am hard to satisfy. & 5 \\
Rule-follower & 5 \\
\hline & 5 \\
\hline
\end{tabular}

and large effect sizes, respectively. Although this issue requires further examination, we suspect that these guidelines are reasonable enough for researchers to find them useful.

Second, the network loading matrix has particularly small cross-loadings, including some loadings that are zero. Many of the small cross-loadings are small not just by traditional factor analysis standards but also partial correlation standards. This is because of the network estimation where many pairwise correlations are shrunk to zero, leaving many nodes not connected to other nodes. Therefore, if a node (component) is not connected to any nodes in another dimension, there is no loading for that node in the dimension. This shrinkage also affects the size of the cross-loadings by making most cross-dimension connections small, resulting in lower loadings.

One peculiar cross-loading is the component set high standards for myself and others with openness to experience. From the loading matrix, it's difficult to discern why this component would be related to openness to experience. The network, however, provides greater insight into this relation, specifically the set high standards for myself and others (Shsfmao) component is connected to the self-assessed intelligence (S-i) and introspective (Int) components (Figure SI4).

\section{Structural consistency}

In the manuscript, we proposed structural consistency as a complementary measure to internal consistency. To compute structural consistency, we must first apply the bootEGA function, which will compute the bootstrap analysis. Once this procedure is completed, the dimStability function can be applied.

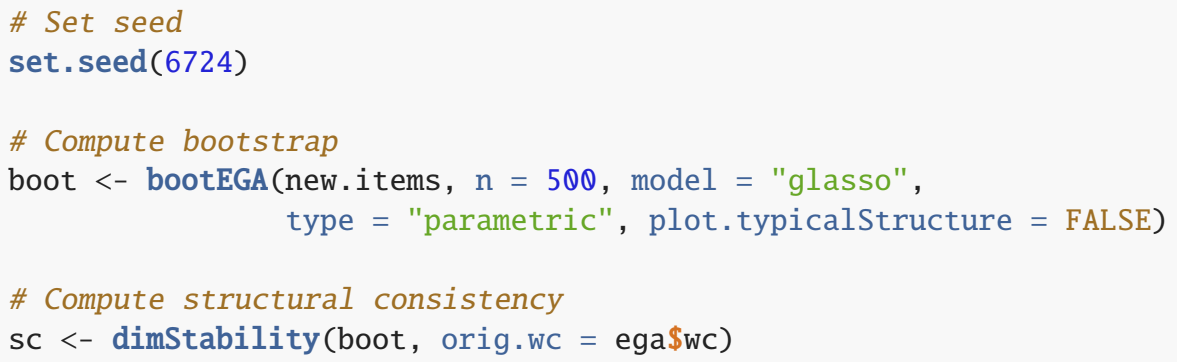


Table SI3. Standardized node strength for each node in each dimension identified by EGA

\begin{tabular}{|c|c|c|c|c|c|}
\hline & 1 & 2 & 3 & 4 & 5 \\
\hline Work hard. & 0.340 & -0.011 & 0.027 & 0.040 & 0.063 \\
\hline Neglect my duties. & -0.314 & 0.047 & 0.003 & 0.007 & -0.115 \\
\hline Perfectionist & 0.241 & 0.045 & 0.000 & 0.000 & 0.052 \\
\hline Orderly & 0.222 & -0.017 & -0.004 & -0.024 & 0.036 \\
\hline Motivated & 0.322 & -0.050 & 0.028 & 0.060 & 0.003 \\
\hline Set high standards for myself and others. & 0.205 & 0.000 & 0.003 & 0.144 & -0.064 \\
\hline Worrier & 0.040 & 0.385 & 0.000 & 0.000 & 0.034 \\
\hline Anxious & 0.015 & 0.405 & -0.048 & -0.076 & 0.006 \\
\hline Low self-esteem & -0.091 & 0.174 & -0.135 & -0.015 & -0.065 \\
\hline Irritable (R) & 0.000 & -0.178 & 0.000 & 0.012 & 0.120 \\
\hline Emotional stability & 0.015 & -0.320 & -0.020 & -0.049 & -0.085 \\
\hline People person & 0.000 & -0.019 & 0.326 & -0.041 & 0.088 \\
\hline Attention-seeking & -0.007 & 0.019 & 0.284 & 0.003 & -0.116 \\
\hline Social-efficacy & 0.038 & -0.094 & 0.341 & 0.048 & 0.014 \\
\hline Laugher & 0.010 & -0.017 & 0.183 & 0.012 & 0.096 \\
\hline Express myself easily. & 0.005 & -0.045 & 0.240 & 0.070 & 0.051 \\
\hline Original ideation & 0.037 & -0.032 & 0.074 & 0.409 & -0.066 \\
\hline Fantasy & -0.044 & 0.030 & 0.020 & 0.302 & -0.048 \\
\hline Introspective & 0.040 & 0.010 & 0.022 & 0.194 & 0.046 \\
\hline Self-assessed intelligence & 0.095 & -0.053 & 0.033 & 0.170 & 0.000 \\
\hline Concerned for others & 0.030 & 0.119 & 0.119 & 0.029 & 0.248 \\
\hline Manipulative & -0.087 & 0.042 & 0.066 & -0.001 & -0.186 \\
\hline Sees good in people & 0.000 & -0.076 & 0.088 & 0.000 & 0.223 \\
\hline Am hard to satisfy. & 0.083 & 0.069 & -0.062 & 0.000 & -0.146 \\
\hline Enjoy being thought of as a normal mainstream person. & 0.000 & 0.001 & 0.012 & -0.115 & 0.126 \\
\hline Rule-follower & 0.123 & 0.011 & -0.042 & -0.054 & 0.230 \\
\hline
\end{tabular}

\# Print structural consistency

sc\$dimensions

Table SI4. Structural consistency values for each dimension

\begin{tabular}{cc}
\hline Dimension & Structural Consistency \\
\hline 1 & 1.000 \\
2 & 1.000 \\
3 & 1.000 \\
4 & 1.000 \\
5 & 0.832 \\
\hline
\end{tabular}

Based on Table SI4, four of the five dimensions have perfect structural consistency. The fifth dimension, agreeableness, appears to be less stable than the others. To get a better understanding of this result, we can plot the item stability statistics: 
Set high standards for myself and others.

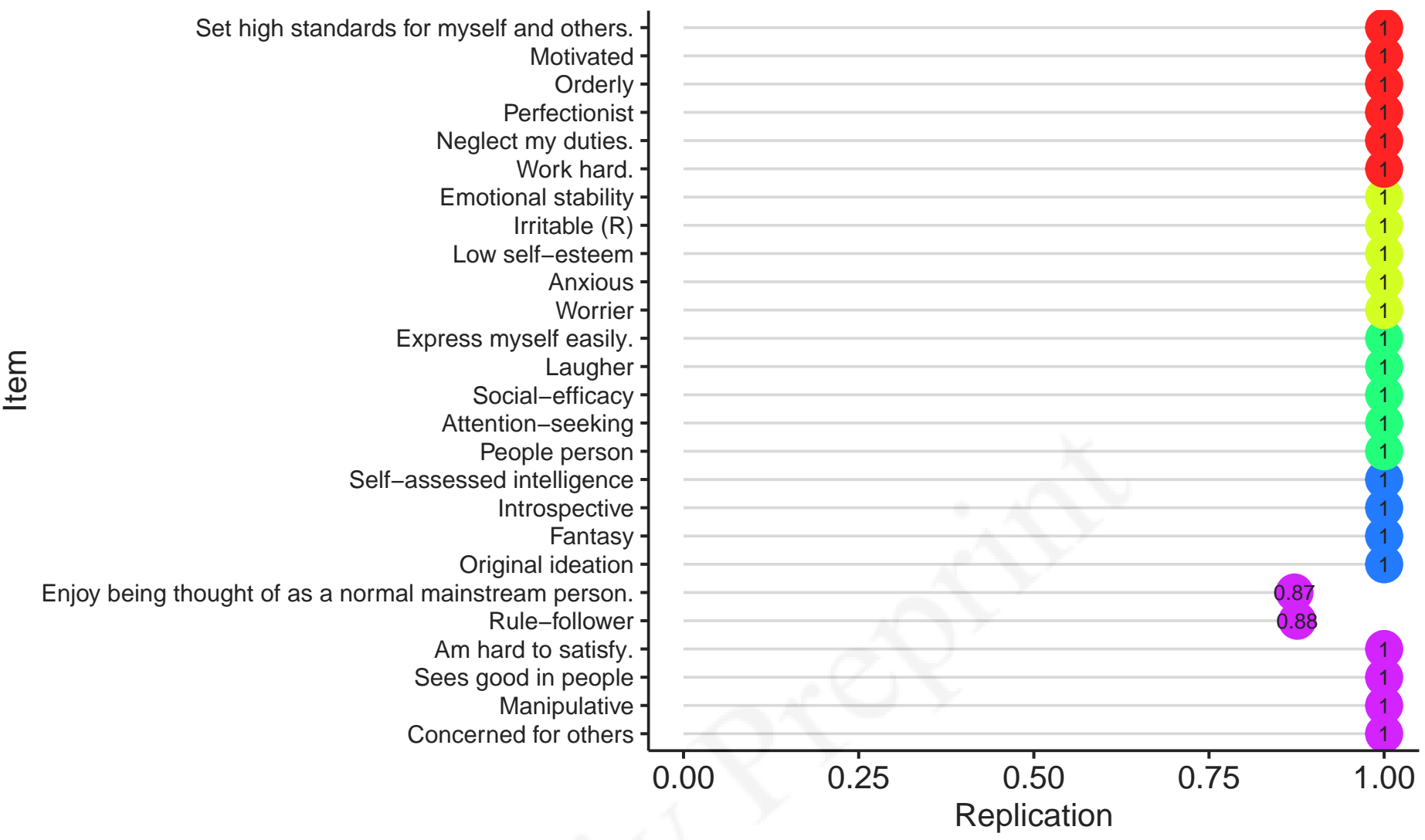

Figure SI5. Each item's replication in the original dimension specified by EGA

The item stability plot reveals that most components within the agreeableness dimension are consistently being identified within their original dimension. To view how these components are positioned across dimensions, the i tems $\$$ i tem. dim. rep object can be viewed:

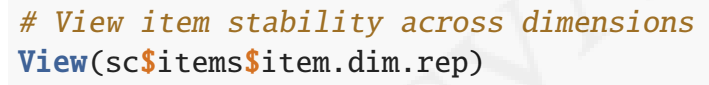

Based on Table SI5, two of the components-Enjoy being thought of as a normal mainstream person. and Rule-follower-are being identified in the fourth dimension or openness to experience about $20 \%$ of the time. This suggests that these components mainly represent the agreeableness domain but also fall within the openness to experience domain. 
Table SI5. Item stability within each dimension

\begin{tabular}{|c|c|c|c|c|c|}
\hline & 1 & 2 & 3 & 4 & 5 \\
\hline Set high standards for myself and others. & 1 & & & & \\
\hline Motivated & 1 & & & & \\
\hline Orderly & 1 & & & & \\
\hline Perfectionist & 1 & & & & \\
\hline Neglect my duties. & 1 & & & & \\
\hline Work hard. & 1 & & & & \\
\hline Emotional stability & & 1 & & & \\
\hline Irritable $(\mathrm{R})$ & & 1 & & & \\
\hline Low self-esteem & & 1 & & & \\
\hline Anxious & & 1 & & & \\
\hline Worrier & & 1 & & & \\
\hline Express myself easily. & & & 1 & & \\
\hline Laugher & & & 1 & & \\
\hline Social-efficacy & & & 1 & & \\
\hline Attention-seeking & & & 1 & & \\
\hline People person & & & 1 & & \\
\hline Self-assessed intelligence & & & & 1 & \\
\hline Introspective & & & & 1 & \\
\hline Fantasy & & & & 1 & \\
\hline Original ideation & & & & 1 & \\
\hline Rule-follower & & & & 0.168 & 0.832 \\
\hline Enjoy being thought of as a normal mainstream person. & & & & 0.168 & 0.832 \\
\hline Am hard to satisfy. & & & & & 1 \\
\hline Sees good in people & & & & & 1 \\
\hline Manipulative & & & & & 1 \\
\hline Concerned for others & & & & & 1 \\
\hline
\end{tabular}




\section{References}

Blondel, V. D., Guillaume, J.-L., Lambiotte, R., \& Lefebvre, E. (2008). Fast unfolding of communities in large networks. Journal of Statistical Mechanics: Theory and Experiment, 2008, P10008. https://doi.org/10.1088/1742-5468/2008/10/P10008

Christensen, A. P. (2020). Towards a network psychometrics approach to assessment: Simulations for redundancy, dimensionality, and loadings (Unpublished doctoral dissertation). University of North Carolina at Greensboro, Greensboro, NC, USA. https://doi.org/10.31234/osf.io/84kgd

Cohen, J. (1992). A power primer. Psychological Bulletin, 112, 155-159. https://doi.org/10.1037/0033-2909.112.1.155

Condon, D. M. (2018). The SAPA personality inventory: An empirically-derived, hierarchically-organized self-report personality assessment model. PsyArXiv. https://doi.org/10.31234/osf.io/sc4p9

Costantini, G., Richetin, J., Preti, E., Casini, E., Epskamp, S., \& Perugini, M. (2019). Stability and variability of personality networks. A tutorial on recent developments in network psychometrics. Personality and Individual Differences, 136, 68-78. https://doi.org/10.1016/j.paid.2017.06.011

Dinic, B. M., Wertag, A., Tomasevic, A., \& Sokolovska, V. (2020). Centrality and redundancy of the Dark Tetrad traits. Personality and Individual Differences, 155, 109621. https://doi.org/10.1016/j.paid.2019.109621

Golino, H., \& Christensen, A. P. (2020). EGAnet: Exploratory Graph Analysis: A framework for estimating the number of dimensions in multivariate data using network psychometrics. Retrieved from https://CRAN.R-project.org/package= EGAnet

Golino, H., \& Epskamp, S. (2017). Exploratory Graph Analysis: A new approach for estimating the number of dimensions in psychological research. PloS ONE, 12, e0174035. https://doi.org/10.1371/journal.pone.0174035

Golino, H., Shi, D., Christensen, A. P., Garrido, L. E., Nieto, M. D., Sadana, R., ... Martinez-Molina, A. (in press). Investigating the performance of Exploratory Graph Analysis and traditional techniques to identify the number of latent factors: A simulation and tutorial. Psychological Methods. https://doi.org/10.1037/met0000255

Gysi, D. M., Voigt, A., de Miranda Fragoso, T., Almaas, E., \& Nowick, K. (2018). wTO: An R package for computing weighted topological overlap and a consensus network with integrated visualization tool. BMC Bioinformatics, $19,392$. https://doi.org/10.1186/s12859-018-2351-7

McCrae, R. R., \& Costa, P. T. (1987). Validation of the five-factor model of personality across instruments and observers. Journal of Personality and Social Psychology, 52, 81-90. https://doi.org/10.1037/0022-3514.52.1.81

Perez, M.-E., \& Pericchi, L. R. (2014). Changing statistical significance with the amount of information: The adaptive $\alpha$ significance level. Statistics \& Probability Letters, 85, 20-24. https://doi.org/10.1016/j.spl.2013.10.018

Pons, P., \& Latapy, M. (2006). Computing communities in large networks using random walks. Journal of Graph Algorithms and Applications, 10, 191-218. https://doi.org/10.7155/jgaa.00185

Revelle, W. (2019). psychTools: Tools to accompany the 'psych' package for psychological research. Evanston, Illinois: Northwestern University. Retrieved from https://CRAN.R-project.org/package=psychTools 


\section{Session Info for Reproducibility}

$\mathrm{R}$ version 3.6.3 (2020-02-29)

Platform: x86_64-w64-mingw32/x64 (64-bit)

Running under: Windows 10 x64 (build 18363)

Matrix products: default

locale:

[1] LC_COLLATE=English_United States. 1252

[2] LC_CTYPE=English_United States. 1252

[3] LC_MONETARY=English_United States. 1252

[4] LC_NUMERIC=C

[5] LC_TIME=English_United States. 1252

attached base packages:

[1] stats graphics grDevices utils datasets methods base

other attached packages:
[1] EGAnet_Q.9.4
psychTools_1.9.12

loaded via a namespace (and not attached):
[1] nlme_3.1-145
fs_1.4.0
RColorBrewer_1.1-2
[4] devtools_2.2.2
backports_1.1.5
usethis_1.5.1
[7] tools_3.6.3
d3Network_Q.5.2.1
nnet_7.3-13
[13] colorspace_1.4-1
prettyunits_1.1.1
[16] npsurv_0.4-0
gridExtra_2.3
[19] tidyselect_1.0.0
qgraph_1.6.5
[22] compiler_3.6.3
htmlTable_1.13.3
[25] cli_2.0.2
[28] desc_1.2.0
labeling_0.3
mvtnorm_1.1-0
pbapply_1.4-2
[34] callr_3.4.3
[37] digest_0.6.25
pbivnorm_0.6.0
jpeg_0.1-8.1
[43] htmltools_0.4.0
sessioninfo_1.1.1
[46] htmlwidgets_1.5.1
rlang_0.4.5
visNetwork_2.0.9
[49] huge_1.3.4
gtools_3.8.2
magrittr_1.5
Rcpp_1.0.4
[55] dplyr_0.8.5
[58] Matrix_1.2-18
[61] fansi_0.4.1
abind_1.4-5
whisker_0.4
plyr_1.8.6
[67] pkgbuild_1.0.6
parallel_3.6.3
[70] grid_3.6.3
splines_3.6.3
pillar_1.4.3
rjson_0.2.20
BDgraph_2.62
codetools_0.2-16
rprojroot_1.3-2
R6_2.4.1
Hmisc_4 . 4-0
withr_2.1.2
processx_3.4.2
mnormt_1.5-6
fdrtool_1.2.15
NetworkToolbox_1.4.1
scales_1.1.0
psych_1.9.12.31
stringr_1.4.0
foreign_0.8-76
pkgconfig_2.0.3
pwr_1.3-0
rstudioapi_Q.11
farver_2.0.3
acepack_1.4.1
Formula_1.2-3
munsell_0.5.0
lifecycle_0.2.0
MASS_7.3-51.5
lavaan_0.6-5
crayon_1.3.4
ps_1.3.2
igraph_1.2.5
ggsignif_0.6.0
pkgload_1.0.2
stats4_3.6.3
$\begin{array}{ll}\text { [88] wT0_1.6.3 } & \text { glue_1.3.2 } \\ \text { [91] latticeExtra_0.6-29 } & \text { remotes_2.1.1 }\end{array}$
1sei_1.2-0
[94] png_0.1-7
vctrs_0.2.4
[97] testthat_2.3.2
[100] assertthat_0.2.1
[103] survival_3.1-11
gtable_0.3.0
ggplot2_3.3.0
data.table_1.12.8
foreach_1.5.0
purrr_0.3.3
xfun_Q 0.12
tibble_3.0.0
glasso_1.11
som_0.3-5.1
memoise_1.1.0
[109] cluster_2.1.0
fitdistrplus_1.0-14
ellipsis_0.3.0 